\title{
Generating Human-like Motion for Robots
}

\author{
Michael J. Gielniak, C. Karen Liu, and Andrea L. Thomaz
}

\begin{abstract}
Action prediction and fluidity are a key elements of human-robot teamwork. If a robot's actions are hard to understand, it can impede fluid HRI. Our goal is to improve the clarity of robot motion by making it more humanlike. We present an algorithm that autonomously synthesizes human-like variants of an input motion. Our approach is a three stage pipeline. First we optimize motion with respect to spatio-temporal correspondence (STC), which emulates the coordinated effects of human joints that are connected by muscles. We present three experiments that validate that our STC optimization approach increases human-likeness and recognition accuracy for human social partners. Next in the pipeline, we avoid repetitive motion by adding variance, through exploiting redundant and underutilized spaces of the input motion, which creates multiple motions from a single input. In two experiments we validate that our variance approach maintains the human-likeness from the previous step, and that a social partner can still accurately recognize the motion's intent. As a final step, we maintain the robot's ability to interact with it's world by providing it the ability to satisfy constraints. We provide experimental analysis of the effects of constraints on the synthesized human-like robot motion variants.
\end{abstract}

\section{INTRODUCTION}

Human-robot collaboration is one important goal of the field of robotics, where a human and a robot work jointly together on shared tasks. Importantly, results indicate that collaboration is improved if the robot exhibits human-like movements [7], [8], [5]. This stems in part from the fact that human-like motion supports natural human-robot interaction by allowing the human user to more easily interpret movements of the robot in terms of goals. This is also called motion clarity.

There is much evidence from human-human interaction, that predicting the motor intentions of others while watching their actions is a fundamental building block of successful joint action [28]. The prediction of action outcomes may be used by the observer to select an adequate complementary behavior in a timely manner [3], contributing to efficient coordination of actions and decisions between the agents in a shared task. Thus, if a robot's motion is such that it is more recognizable it will better afford anticipation and intention prediction, and will contribute to improving the human-robot collaboration.

Hence, the goal of our work is to produce algorithmic solutions for generating human-like motion on a social robot.

M. Gielniak is a postdoctoral researcher, Georgia Institute of Technology, Atlanta, GA, 30332, USA mgielniak3@gatech. edu

C. Karen Liu is with the College of Computing, Georgia Institute of Technology, Atlanta, GA, 30332, USA karenliu@Cc.gatech.edu

A. Thomaz is with the College of Computing, Georgia Institute of Technology, Atlanta, GA, 30332, USA athomaz@cc.gatech.edu
Our approach divides the process of generating human-like motion into three components:

1) Spatio-temporal coordination: The first aspect of our algorithm takes an input motion and makes it more human-like by applying a spatio-temporal optimization, emulating the coordinated effects of human joints that are connected by muscles.

2) Variance: The second aspect of our algorithm adds variance. Humans never move in the same way twice, so variance in and of itself can contribute to making motion human-like.

3) Constraints: The final aspect of our algorithm makes the new human-like variant of the input motion practically applicable in the face of environmental constraints.

The majority of existing motion generation techniques for social robots do not produce human-like motion. For example, retargeting human motion capture data to robots does not produce human-like motion for robots because the degreesof-freedom differ in number or location on the kinematic structures of robots and humans. The projection of motion causes information to be lost, and the human motion can look much different (often quite poor) on the robot kinematic hierarchy. Also, in the rare instances when retargeting human motion to robots works well, it produces only one motion trajectory, rather than a variety of trajectories, which makes the robot move in a very repetitive way.

We present an algorithm for the generation of an infinite number of human-like motion variants from a single exemplar. Through a series of experiments with human participants, we provide evidence that these variants are human-like, increase motion recognition, and respect the task (i.e. are quantitatively and qualitatively classified as the same motion type as the input exemplar). Furthermore, this algorithm can be combined with joint and Cartesian-space constraints to ensure that position, velocity, and acceleration are satisfied. These constraints will enable social robots to accomplish sophisticated tasks such as synchronization with human partners.

\section{RELATED WORK}

\section{A. Human-like Motion in Robotics}

A fundamental problem with existing techniques that generate robot motion is data dependence. For example, a very common technique is to build a model for a particular motion from a large number of exemplars [18], [30], [22]. Ideally, the robot could observe one (potentially bad) exemplar of a motion and generalize it to a more human-like counterpart. 
Dependence on large quantities of data is often an empirical substitute for a more principled approach. For example, rapidly exploring random tree (RRT) based methods offer no guarantees of human-like motion, but relies upon a database of natural or human-like motions to bias the solution towards realism. Searching the database to find a motion similar to the RRT-generated trajectory is a bottleneck for online planning, which can affect algorithm runtime [33].

Other techniques rely upon empirical relationships derived from the data to constrain robot motion to appear more human-like. This includes criteria such as joint comfort, movement time, jerk, [12], [6], and human pose-to-target relationships [16], [31]. When motion capture data is used, often timing is neglected, which causes robot motion to occur at unrealistic and non-human velocities [2], [1].

\section{B. Human-like Motion in Computer Graphics}

Motion techniques developed for cartoon or virtual characters cannot be immediately applied to robots because fundamental differences exist in the real-world. The extent to which techniques designed for graphics can be applied to robots depends on the assumptions made for a particular technique. Constraints such as torque or velocity limits of actual hardware often cause motion synthesized for virtual characters to look poor on a robot, even when the motion looks good on a virtual model because less strict limits exist for the virtual world.

Data-dependent techniques such as annotated databases of human motion are also very common in computer animation [4], [23]. These suffer from the previously mentioned insufficiencies, such as lack of variance, because variance is limited by the size of the database.

Optimization is a common technique used to generate human-like motion or change human motion retargeted to a virtual character in the presence of new constraints. The former works when human data profiles (e.g. human momentum [20], minimum jerk [6]) are used, and the latter works well when a second, lower-dimensional model exists [24]. The disadvantage is that the human-recorded trajectory might not be applicable or extensible to other scenarios, which results in the collection of larger quantities of data to work well for a specific motion or scenario. And in the case of spacetime optimization, where constraints are solved on a simpler model and motion is projected back to a more complex model, the manual creation of the second, low-dimensional model is a disadvantage. In general, optimization is computationally costly for high numbers of DOFs, sensitive to initial conditions, and nonlinear, which hinders solution convergence.

\section{Existing Human-like Motion Metrics}

Human perception is often the metric for quality in robot motion. By modulating physical quantities like gravity in dynamic simulations from normal values and measuring human perception sensitivity to error in motion, studies can yield a range of values for the physical variables that are below the measured perceptible error threshold (i.e.

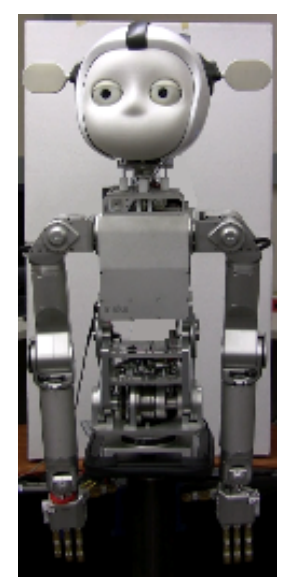

Fig. 1. The robot platform used in this research is an upper-torso humanoid robot called Simon.

effectively equivalent to the human eye) [26], [32]. These techniques are valuable as both synthesis and measurement tools. However, the primary problem with this type of metric is dependency on human input to judge acceptable ranges. Results may not be extensible to all motions without testing new motions with new user studies because these metrics depend upon quantifying the measurement device (i.e. human perception).

Classifiers have been used to distinguish between natural and unnatural movement based on human-labeled data. If a Gaussian mixture model, hidden Markov model, switching linear dynamic system, naive Bayesian, or other statistical model can represent a database of motions, then by training one such model based on good motion-capture data and another based on edited or noise-corrupted motion capture data, the better predictive model would more accurately match the test data. This approach is inspired by the theory that humans are good classifiers of motion because they have witnessed a lot of motion. However, data dependence is the problem, and retraining is necessary when significantly different exemplars are added [27].

In our literature search, we found no widely accepted metric for human-like motion in the fields of robotics, computer animation, or biomechanics. By far, the most common validation efforts rely upon subjective observation and are not quantitative. For example, the ground truth estimates produced by computer animation algorithms are evaluated and validated widely based on qualitative assessment and visual inspection. Other forms of validation include projection of motion onto a 2-D or 3-D virtual character to see if the movements seem human-like [21]. Our work presents a metric for human-like motion that is quantitative.

\section{RESEARCH PLATFORM}

The robot platform used in our research is an uppertorso humanoid robot called Simon. It has two controllable DOFs on the torso, seven DOFs per arm, four per hand, two per ear, three eye DOFs, and four neck degrees-offreedom. The robot operates on a dedicated ethercat network 
coupled with a real-time PC operating at a frequency of $1 \mathrm{kHz}$. To maintain highly accurate joint angle positions, the hardware is controlled with PID gains of very high magnitude, providing rapid transient response.

\section{ALGORITHM}

In this section we detail each of the three components of our approach to generating human-like motion for robots. Each of these components is one piece in a pipeline. The first step is our algorithm for optimizing spatiotemporal correspondence. The next component is an optimization to add variance while remaining consistent with the intent of the original motion and obeying constraints of the task. The final component in the pipeline is an algorithm for generating transitions between these human-like motion trajectories, such that this pipeline can produce continuous robot motion.

\section{A. Human-like Optimization}

Due to muscles that connect degrees-of-freedom, the trajectories of proximal DOFs on a human exhibit coordination or correspondence, meaning that motion of one DOF influences the others connected. However, robots have motors, and the trajectories of proximal degrees-of-freedom do not influence each other. In theory, if the effect of trajectories influencing each other is created for proximal robot DOFs by increasing the amount of spatial (SC) and temporal coordination (TC) for robot motion, it should become more human-like.

The spatiotemporal correspondence problem has already been heavily studied and analyzed mathematically for a pair of trajectory sets, where there is a one-to-one correspondence between trajectories in each set (e.g. two human bodies, both of which have completely defined and completely identical kinematic hierarchies and dynamic properties) [10], [15]. Given two trajectories $\mathrm{x}(\mathrm{t})$ and $\mathrm{y}(\mathrm{t})$, correspondence entails determining the combination of sets of spatial $(a(t))$ and temporal $(b(t))$ shifts that map two trajectories onto each other. In the absence of constraints, the temporal and spatial shifts satisfy the equations in 1 , where reference trajectory $\mathrm{x}(\mathrm{t})$ is being mapped onto $\mathrm{y}(\mathrm{t})$.

$$
\begin{aligned}
y(t) & =x\left(t^{\prime}\right)+a(t) \\
t^{\prime} & =t+b(t)
\end{aligned}
$$

where,

$$
\begin{aligned}
& x(t)=\text { first reference trajectory } \\
& y(t)=\text { second reference or output trajectory } \\
& a(t)=\text { set of time-dependent spatial shifts } \\
& b(t)=\text { set of time-dependent temporal shifts } \\
& t \quad=\text { time } \\
& t^{\prime} \quad=\text { temporally shifted time variable }
\end{aligned}
$$

The correspondence problem is ill-posed, meaning that the set of spatial and temporal shifts is not unique. Therefore, a metric is often used to define a unique set of shifts.
Spatial-only metrics, which constitute the majority of "distance" metrics, are insufficient when data includes spatial and temporal relationships. Spatiotemporal-Isomap (ST-Isomap) is a common algorithm that takes advantage of STC in data to reduce dimensionality. However, the geodesic distancebased algorithm at the core of ST-Isomap was not selected as the candidate metric due to manual tuning of thresholds and operator input required to cleanly establish correspondence [14]. Another critical requirement for a metric is nonlinearity, since human motion data is nonlinear.

Our algorithm begins with the assumption that an input exemplar motion exists, for which human-like variants should be generated. To emulate the local coupling exhibited in human DOFs (e.g. ball-and-socket joints, muscular interdependence) on an anthropomorphic robot, which typically has serial DOFs, we optimize torque trajectories from the original motion according to the metric shown in Equation 2 based on parent and children degrees-of-freedom, in the hierarchical anthropomorphic chain.

$$
\begin{array}{r}
C_{\left(d_{s}, d_{t}\right)}(S, T, r)= \\
\frac{\sum_{l=1}^{T} \sum_{j=1}^{T} \sum_{g=1}^{S} \sum_{h=1}^{S} \Theta\left(r-\left\|V_{l}^{g}-V_{j}^{h}\right\|\right)}{(T-1) T(S-1) S} \\
K_{2}(S, T, r)= \\
\ln \left(\frac{C_{d_{s}, d_{t}}(S, T, r)}{C_{d_{s}, d_{t}+1}(S, T, r)}\right)+\ln \left(\frac{C_{d_{s}, d_{t}}(S, T, r)}{C_{d_{s}+1, d_{t}}(S, T, r)}\right)
\end{array}
$$

where,

$\Theta(\ldots)=$ Heaviside step function

$V_{i}^{k}=\left[w_{i}^{k}, \ldots, w_{i}^{k+d_{s}-1}\right]$, spatiotemporal delay vectors

$w_{i}^{k}=\left[v_{i}^{k}, \ldots, v_{i+d_{t}-1}^{k}\right]$, time delay vectors

$v_{i}^{k} \quad=$ element of time series trajectory for actuator $k$ at time index $i$

$d_{s} \quad=$ spatial embedding dimension

$d_{t} \quad=$ temporal embedding dimension

$S \quad=$ number of actuators

$T \quad=$ number of motion time samples

$r \quad=$ correspondence threshold

Before continuing, it might be helpful to provide some insight into the metric in Equation 3. It was originally developed for use in chaos theory to measure rate of system state information loss between measurements of the same signal as a function of time [25]. Chaotic signals will yield different values upon successive measurements, and the metric is used to measure similarity between two measurements of the same signal. Our insight is that the same metric can be used on two separate deterministic signals (e.g. trajectories) as a similarity metric to determine how similar these trajectories in terms of both space and timing. The metric discretizes the state space (i.e. torque space) into d-dimensional segments of size $r^{d}$, where $d$ can be either the spatial or temporal embedding dimension. Temporal delay vectors are used as 
part of the comparisons between the discretized segments of each trajectory.

The $K_{2}$ metric presented in Equation 3 constrains the amount of trajectory modulation in three parameters: $r, S$, and T. $r$ and can be thought of as a resolution or similarity threshold. Every spatial or temporal pair below this threshold would be considered equivalent and everything above it, non-equivalent and subject to modulation. We empirically determined a 0.1 N.m. threshold for $r$ on the Simon robot hardware.

Based upon the assumption of a predefined input motion, temporal extent, $T$, varies based on the sequence length for a given motion. And, to emulate the local coupling exhibited in human DOFs on an anthropomorphic robot, the spatial parameter, $S$, is set at a value that optimizes only based upon parent and children degrees-of-freedom, in the hierarchical anthropomorphic chain.

When modulating trajectories, the optimization begins at the "root" DOF (typically a rotationally motionless DOF, like the pelvis), and it extends outward toward the fingertips. For Simon, this "root" DOF represents the rigid mount to the base. In other robots, the DOF nearest to the center-of-gravity is a logical place to begin the optimization, which can extend outward along each separate DOF chain. Any optimization that accepts a cost function can be used (e.g. optimal control, dynamic time warping), and the metric presented in Equation 3 would be substituted for the cost function in the problem definition.

\section{B. Variance Optimization}

Once the process of inducing coupling between DOFs that are locally proximal on the hierarchy is complete, the output trajectory of the human-like optimization is used as the reference trajectory for a second optimization to add variance and satisfy constraints. The objective of this second optimization is to produce human-like variants without corrupting the original motion intent. The second optimization yields a biased torque that optimally preserves the characteristics of the input motion encoded in the cost function and any jointspace constraints. This biased torque is projected to the null space of Cartesian constraints to ensure that they are preserved. The resultant torque stochastically produces motion that is visually different from the input while maintaining constraints.

The core of our algorithm computes a time-varying multivariate Gaussian that has shaped covariance matrices, $\mathcal{N}\left(0, S_{t}^{-1}\right)$, constraint projection matrices, $P_{t}$, and a feedback control policy, $\Delta u_{t}=-K_{t} \Delta x_{t}$, where $K_{t}$ represents the feedback control gain. The characteristics of the input motion are represented by the Gaussian and the feedback policy, while the Cartesian constraints are preserved by the projection matrices.

Variation for an input motion is generated online by applying the following operations at each time step, which are explained in detail in subsequent sections. As closely as possible, we maintain the variable conventions for optimal control, so that notations are familiar from control theory.
1) Shape the torque covariance matrix for a Gaussian and draw a random sample: $\Delta x_{t} \sim \mathcal{N}\left(0, S_{t}^{-1}\right)$

2) Preserve joint space constraints by appropriately defining two weight matrices: $Q_{t}$ and $R_{t}$, which scale the relative importance of the state and control terms, respectively, in the optimization.

3) Compute the corresponding control force via the feedback control policy: $\Delta u_{t}=-K_{t} \Delta x_{t}$

4) Project the control force to enforce Cartesian constraints: $\Delta u_{t}^{*}=P_{t} \Delta u_{t}$

5) Apply the input motion and projected torque, $\bar{u}_{t}+\Delta u_{t}^{*}$, as the current control force

1) Shaping Torque Noise: Since the human-like optimization performs correspondence on torque trajectories, forward dynamics is used to compute the time varying set of joint angles that comprise the motion. For the second optimization, the time-varying sequence of joint angles, $q_{t}$, which were formed from the motion output from the humanlike optimization are constructed into a reference state trajectory, $\bar{x}$, along with $\dot{q}_{t}$, the time-varying joint velocities. A reference control trajectory, $\bar{u}$, which consists of joint torques is also formed using the direct output from the human-like optimization.

The goal of the variance optimization is to minimize the state and control deviation from the reference trajectory, subject to discrete-time dynamic equations, as shown in Equation 4.

$$
\begin{gathered}
\min _{x, u} \frac{1}{2}\left\|x_{N}-\bar{x}_{N}\right\|_{S_{N}}^{2}+\ldots \\
\sum_{t=0}^{N-1} \frac{1}{2}\left(\left\|x_{t}-\bar{x}_{t}\right\|_{Q_{t}}^{2}+\left\|u_{t}-\bar{u}_{t}\right\|_{R_{t}}^{2}\right) \\
\text { subject to } x_{t+1}=f\left(x_{t}, u_{t}\right)
\end{gathered}
$$

For an optimal control problem (Equation 4), it is convenient to define an optimal value function, $v\left(x_{t}\right)$, which measures the minimal total cost of the trajectory from state, $x_{t}$. Evaluation of the optimal value function defines the optimal action at each state. It can be written recursively, using the shorthand notation, $\|x\|_{Y}^{2}=x^{T} Y x$, in Equation 5.

$$
v\left(x_{t}\right)=\min _{u} \frac{1}{2}\left(\left\|x_{t}-\bar{x}_{t}\right\|_{Q_{t}}^{2}+\left\|u_{t}-\bar{u}_{t}\right\|_{R_{t}}^{2}\right)+v\left(x_{t+1}\right)
$$

Our key insight is that the shape of this value function reveals information about the tolerance of the control policy to perturbations. With this, we can choose a perturbation that causes minimal disruption to the motion intent while inducing visible variation to the reference motion.

Both human and robot motion are nonlinear, and the optimal value function is usually very difficult to solve for a nonlinear problem. We approximate the full nonlinear dynamic tracking problem with linear quadratic regulator (LQR), which has a linear dynamic equation and a quadratic cost function. From a full optimal control problem, we linearize the dynamic equation around the reference trajectory and substitute the variables with the deviation from the reference, $\Delta x$ and $\Delta u$. 


$$
\begin{array}{r}
\min _{\Delta x, \Delta u} \frac{1}{2}\left\|\Delta x_{N}\right\|_{S_{N}}^{2}+\sum_{t=0}^{N-1} \frac{1}{2}\left(\left\|\Delta x_{t}\right\|_{Q_{t}}^{2}+\left\|\Delta u_{t}\right\|_{R_{t}}^{2}\right) \\
\text { subject to } \Delta x_{t+1}=A_{t} \Delta x_{t}+B_{t} \Delta u_{t}
\end{array}
$$

where $A_{t}=\left.\frac{\partial f}{\partial x}\right|_{\bar{x}_{t}, \bar{u}_{t}}, B_{t}=\left.\frac{\partial f}{\partial u}\right|_{\bar{x}_{t}, \bar{u}_{t}}$.

$S_{N}, Q_{t}$, and $R_{t}$ are positive semidefinite matrices that indicate the time-varying weights between different objective terms. We will discuss how these terms are computed to satisfy joint-space constraints later.

The primary reason to approximate our problem with a time-varying $L Q R$ formulation is that the optimal value function can be represented in quadratic form with timevarying Hessians.

$$
v\left(\Delta x_{t}\right)=\frac{1}{2}\left\|\Delta x_{t}\right\|_{S_{t}}^{2}
$$

where the Hessian matrix, $S_{t}$, is a symmetric matrix.

The result of linearizing about the reference trajectory is that at time step $t$, the optimal value function is a quadratic function centered at the minimal point $\bar{x}_{t}$. Therefore, the gradient of the optimal value function at $\bar{x}_{t}$ vanishes, while the Hessian is symmetric, positive semidefinite, and measures the curvatures along each direction in the state domain. A deviation from $\bar{x}_{t}$ along a direction with high curvature causes large penalty in the objective function and is considered inconsistent with the human-like motion. For example, the perturbation in the direction of the first eigenvector (the largest eigenvalue) of the Hessian induces the largest total cost of tracking the reference trajectory.

We induce more noise in the dimensions consistent with tracking the human-like input motion by shaping a zero-mean Gaussian with a time-varying covariance matrix defined as the inverse of the Hessian, $\mathcal{N}\left(0, S_{t}^{-1}\right)$. The matrices $S_{t}$ can be efficiently computed by the Riccati equation, shown in Equation 8, which exploits backward recursive relations starting from the weight matrix at the last time step, $S_{N}$. We omit the subscript $t$ on $A, B, Q$, and $R$ for clarity (detailed derivation in [19]).

$$
\begin{gathered}
S_{t}=Q+A^{T} S_{t+1} A- \\
A^{T} S_{t+1} B\left(R+B^{T} S_{t+1} B\right)^{-1} B^{T} S_{t+1} A
\end{gathered}
$$

Solving this optimization defines how to shape the covariance matrices for all time so that variance can be added in dimensions consistent with the input motion, which in our case is the output of the human-like optimization step. But, we have not yet described how to create variance from the human-like input motion using these shaped covariance matrices.

2) Preserving Joint-Space Constraints: Before we can solve the variance optimization, it is necessary to define the cost matrices in the optimization (i.e. $S_{N}, Q_{t}$, and $R_{t}$ ) appropriately so that joint-space constraints are preserved. The cost matrices define how closely the optimization output matches values of joint positions, velocities, or control torques. Since the weight matrices are time-varying, joint-space constraints are also time-varying. High weights at a specific time will generate variants that are biased toward preservation of that specific value at the appropriate time in all variants.

The cost weight matrices, $S_{N}, Q_{t} R_{t}$, can be selected manually based on prior knowledge of the input motion and control. Intuitively, when a joint or actuator is unimportant we assign a small value to the corresponding diagonal entry in these matrices. Likewise, when two joints are moving in synchrony, we give them similar weights.

$Q_{t}$ is the weight matrix for the state (i.e. joint position and velocity). Thus, to preserve joint-space constraints, the respective of weights of the desired DOFs in the $Q_{t}$ matrix are increased so there is a very high cost of deviation from the original trajectory. Similarly, $R_{t}$ is the weight matrix for joint torques. To preserve the control from the input motion, high weights for the desired DOFs at the specific time instants will preserve this control torque in all the output variants at the respective time instants. Joint-space constraints do not need to be specified outside of the time ranges for which they need to be satisfied to ensure they are met at the desired times. Since the optimal control problem is solved backward and sequentially, the formulation takes care of minimizing variance around temporally-local constraints so that motion remains smooth and human-like in all output variants.

$Q$ and $R$ in theory can vary over time, but in the absence of joint-space constraints, most practical controllers hold $Q$ and $R$ fixed to simplify the design process.

We propose a method to automatically determine the cost weights based on coordination in the reference trajectory. The weights for any DOFs constrained in joint-space should overwrite the values output from this automatic algorithm. We apply Principal Component Analysis (PCA) on the reference motion $\bar{x}$ and on the reference control $\bar{u}$ to obtain respective sets of eigenvectors $E$ and eigenvalues $\Sigma$ (in diagonal matrix form). The weight matrix for motion can be computed by $Q=E \Sigma E^{T}$. By multiplying $\Delta x$ on both sides of $Q$, we effectively transform the $\Delta x$ into eigenspace, scaled by the eigenvalues. As a result, $Q$ preserves the coordination of joints in the reference motion, scaled by their importance. $R$ can be computed in the same way. In our implementation, we set $S_{N}$ equal to $Q$.

3) Computing the Control Force that Corresponds to the Shaped Gaussian Sample: We are ready to solve the variance optimization, and thus, we describe how to create variations of the input motion using these shaped covariance matrices.

A random sample, $\Delta x_{t}$, is drawn from the Gaussian $\mathcal{N}\left(0, S_{t}^{-1}\right)$, which indicates deviation from the reference state trajectory, $\bar{x}_{t}$. Directly applying this state deviation to joint angle trajectories will cause vibration. Instead, we induce noise in torque space via the feedback control policy derived from LQR, $\Delta u_{t}=-K_{t} \Delta x_{t}$. In our discrete-time, finite-horizon formulation, the feedback gain matrix, $K_{t}$, is a $m \times 2 n$ time-varying matrix computed in closed-form from Equation 9. 


$$
K_{t}=\left(R+B^{T} S_{t+1} B\right)^{-1} B^{T} S_{t+1} A
$$

Occasionally, the Hessians of the optimal value function become singular. In this case, we apply singular value decomposition on the Hessian to obtain a set of orthogonal eigenvectors $E$ and eigenvalues $\sigma_{1} \cdots \sigma_{n}$ (because $S_{t}$ is always symmetric). For each eigenvector $e_{i}$, we define a one-dimensional Gaussian with zero mean and a variance inversely proportional to the corresponding eigenvalue: $N_{i}\left(0, \frac{1}{\sigma_{i}}\right)$. For those eigenvectors with zero eigenvalue, we simply set the variance to a chosen maximal value (e.g., the largest eigenvalue of the covariance matrix in the entire sequence). The final sample $\Delta x_{t}$ is a weighted sum of eigenvectors: $\Delta x_{t}=\sum_{i} w_{i} e_{i}$, where $w_{i}$ is a random number drawn from $N_{i}$.

4) Preserving Cartesian Constraints: In addition to maintaining characteristics of the input motion, we also want variance that adheres to Cartesian constraints. At each iteration, we define a projection matrix $P_{t}$, as in Equation 10, that maps the variation in torque, $\Delta u_{t}$, to the appropriate control torque that does not interfere with the given kinematic constraint. The Jacobian of the constraint, $J_{t}=\frac{\partial p}{\partial q_{t}}$, maps the Cartesian force required to maintain a point, $p$, to a joint torque, $\tau=J_{t}^{T} f$.

$$
P_{t}=I-J_{t}^{T} \bar{J}_{t}^{T}
$$

where $\bar{J}_{t}$ is one of the many pseudo inverse matrices of $J$. We use the "dynamically consistent generalized inverse", which is shown in Equation 11 [29].

$$
\bar{J}_{t}^{T}=\Lambda_{t} J_{t} M_{t}^{-1}
$$

where $\Lambda_{t}$ and $M_{t}$ are the current inertia matrix in Cartesian space and in joint space.

When we apply the projection matrix $P_{t}$ to a torque vector, it removes the components in the space spanned by the columns of $\bar{J}_{t}$, where the variation will directly affect the satisfaction of the constraint. Consequently, the final torque variation $\Delta u_{t}^{*}=P_{t} \Delta u_{t}$ applied to the robot will maintain the Cartesian constraints. Our algorithm can achieve a variety of Cartesian constraints, such as holding a cup, gazing or pointing at an object.

5) Generating Constrained Variants: The final output after both optimizations is realized by applying the humanlike motion torques, $\bar{u}_{t}$, and the projected torque, $\Delta u_{t}^{*}$ to the robot actuators to generate a single variant of the human-like motion that respects both joint and Cartesianspace constraints. Each new series of random samples drawn from the Gaussian with shaped covariance matrices for all time $t$ will produce a new human-like variant that respects constraints (after the projection). No calculations other than the projection need to be computed after the two optimizations are solved the first time, provided that the time-varying sequence of Hessians and human-like torques are stored in memory for a given input motion.

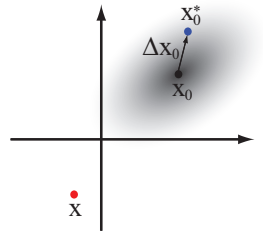

(a)

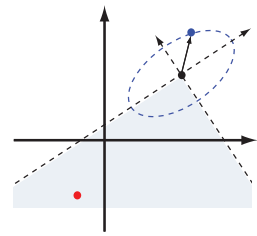

(b)

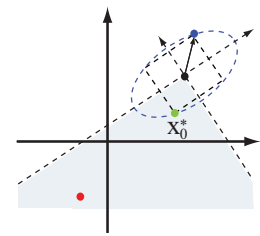

(c)
Fig. 2. (a): Setting the transition-to pose to $x_{0}^{*}=\bar{x}_{0}+\Delta x_{0}$, can generate an awkward transition when $x_{0}^{*}$ is further away from $x$ than from $\bar{x}_{0}$. (b): States with the same likelihood as $\bar{x}_{0}+\Delta x_{0}$ form a hyper-ellipsoid. (c): $\Delta x_{0}$ defines a hypercube aligned with the eigenvectors of the covariance matrix. We pick $x_{0}^{*}$ as the corner that lies in the quadrant of $x$.

\section{Creating Continuous Motion}

Since robots require the ability to move continuously, we describe how our algorithm can be used to continuously produce varied, human-like motion that respects constraints. We demonstrate that it is possible to transition to the next desired motion from a wide range of states. Furthermore, to make the transition reflect natural variance, we stochastically select the starting state of the next motion, called the transition-to pose, online so that it contains variance. We call our transition motions nondeterministic transitions because between the same two motions, different transitions are produced each time.

Once the next motion is selected, our algorithm determines a transition-to pose, $x_{0}^{*}$, via a stochastic process, so that the robot does not always predictably transition to the first state of the next motion. This can be viewed as variance from the first state of the next motion, $\bar{x}_{0}$. We reuse the Gaussian, $\mathcal{N}\left(0, S_{0}^{-1}\right)$, which was computed for the next motion to get a random sample, $\Delta x_{0}$. If we directly set the transition-to pose to $x_{0}^{*}=\bar{x}_{0}+\Delta x_{0}$, it could generate an awkward transition when $x_{0}^{*}$ is further away from the current state $x$ than from $\bar{x}_{0}$ (Figure 2 (a)).

To overcome this issue, we account for the current state, which is denoted as $x$ in Figure 2, when selecting the transition-to pose, $x_{0}^{*}$. Because $\Delta x_{0}$ is drawn from a symmetric distribution, states with the same likelihood form a hyperellipsoid. To bias $x_{0}^{*}$ toward the $x$, we need to pick a state from this hyper-ellipsoid that lies in the same quadrant in the coordinates defined by the eigenvectors of the covariant matrix, $S_{0}^{-1}$, (Figure 2 (b)). To speed up computation, we use $\Delta x_{0}$ to define a hypercube aligned with the eigenvectors. We select $x_{0}^{*}$ to be the corner within the same quadrant as $x$ (Figure 2 (c)).

After determining the transition-to pose, we use spline interpolation on the state and PID-tracking to move the robot to this pose from the current pose. This works well because the transition-to pose is both consistent with the next motion and biased toward the current state.

\section{HYPOTHESES}

In the remainder of this paper, we evaluate the impact and effectiveness of different aspects of this pipeline for generating human-like motion. The respective hypotheses are 
divided up into categories based upon where the input motion is along the process of being transformed into human-like, constrained variants.

\section{A. Human-like Optimization}

Our hypotheses are broken down into four distinct groups based upon topic. The first two hypotheses are based on our expectations for the human-like optimization.

- H1: The human-like optimization increases motion recognition. Thus, motion that has been optimized with respect to spatiotemporal correspondence will be easier for people to correctly identify intent (i.e. the task).

- H2: Spatial and temporal correspondence separately are better metrics for human-likeness than composite spatio-temporal correspondence (STC).

When trajectories that were developed on one kinematic hierarchy (e.g. human) are applied to move another hierarchy (e.g. robot) that is kinematically or dynamically different (e.g. due to lack of DOF correspondence) motion trajectory data is lost. However, motion trajectory data can also be lost because the data is corrupted (e.g. insufficent sample rates in recording equipment). These are examples of using motion data in less-than-ideal conditions. The next three hypotheses are based upon the effects that we expect the human-like optimization to induce upon motion when used in less-thanideal conditions. For rigor, we also include and test the ideal conditions. These hypotheses arise from our expectation that improving trajectory coordination from proximal motors offsets problems that arise due to DOF correspondence.

- H3: The human-like optimization makes robot motion more human-like for imperfect (i.e. non-human-like) models. Thus, information lost due to DOF correspondence is regained by proximal DOF optimization with respect to spatiotemporal motor coordination.

- H4: The human-like optimization has no effect when motion is projected onto a perfect model (i.e. when data-captured human and target model are sufficiently similar).

- H5: The human-like optimization makes motion trajectories more human-like for imperfect data (i.e. when data loss exists). As more data is lost, the human-like optimization produces less optimal results.

\section{B. Variance Optimization}

The next two hypotheses are relevant to the effect that the variance optimization has on the output of the humanlike optimization. Since they are serial optimizations, ideally we want all our generated variants to be at least as humanlike as the motion from which the variants are generated. Furthermore, the variance optimization should not corrupt the original intent of the motion (i.e. if the input is classified by observers as a wave, all variants should also be classified as a wave). We want to test both the quality of the variance optimization output, i.e. human-likeness, and the more fundamental property that our variance optimization produces variants (recognized and labeled same as the input motion).
- H6: The variance optimization preserves humanlikeness.

- H7: The variance optimization preserves intent in the original motion.

\section{Constraints}

Our final three hypotheses test the effect of applied constraints on human-likeness and variance. The effects of constraints are tested in terms of both number of constraints and proximity of constraints to degrees-of-freedom.

- H8: As the number of applied constraints increases, motion becomes less coordinated and less human-like.

- H9: As the number of applied constraints increases, variance decreases.

- H10: Closer to location of application of a Cartesian constraint, the variance optimization produces less variance due to a smaller null space.

\section{EXPERIMENT 1: MIMICKING}

The purpose of our first experiment is to quantitatively support that increasing spatiotemporal correspondence of distributed actuators synthesizes motion that is more humanlike. Since human motion exhibits spatial and temporal correspondence, robot motion that is more coordinated with respect to space and timing should be more human-like. Thus, we hypothesize that motor coordination as produced by $\mathrm{SC}$ and $\mathrm{TC}$ is a metric for human-like motion.

Testing this hypothesis requires a quantitative way to measure human-likeness. Distance measures between human and robot motion variables (e.g. torques, joint angles, joint velocities) in joint space cannot be used without retargeting (i.e. a domain change) due to the DOF correspondence problem. Thus, we designed an experiment based on mimicking.

In short, people are asked to mimick robot motions created by different motion synthesis techniques, and the technique that produced motions that humans were able to mimic the "best" (to be defined later) is deemed the technique that generates the most human-like motion. This experiment assumes that a human-like motion should be easier for people to mimic accurately, and awkward, less natural motions should be harder to mimic.

\section{A. Experimental Design}

In this experiment human motion was measured with a Vicon motion capture system. We examined differences in people's mimicking performance when they attempted to mimic the following three types of stimulus motion:

- Original Human $(\mathrm{OH})$ : Twenty motions captured from one male human, displayed on a virtual human model that precisely matches the marker data (i.e., no retargeting takes place).

- Original Retargeted (OR): The “original human" motions were retargeted to the Simon hardware using a standard retargeting process [11].

- Original Coordinated (OC): The "original retargeted" motions were then coordinated using the human-like optimization. 
These three different "original" datasets were created before the experiment. The twenty motions used in the experiment included common social robot gestures both unconstrained and constrained, such as waving and objectmoving, but also nonsense motions like "air-guitar." The full set of the motions used was: shrug, one-hand bow, twohand bow, scan the distance, worship, presentation, air-guitar, shucks, bird, stick 'em up, cradle, take cover, sneeze, clap, look around, wave, beckon, move object, throw, and call/yell. The latter six motions were constrained with objects for gesture directionality or manipulation, such as a box placed in a certain location to wave toward. When participants were asked to mimic such motions, these constraints were given to them to facilitate ability to mimic accurately. For all participants, the constraint locations and the standing position of the participant were identical. When constraints were given, they were given in all experimental conditions to avoid bias. The air-guitar motion was unconstrained because when humans perform an air-guitar motion, they do not have a guitar in their hands.

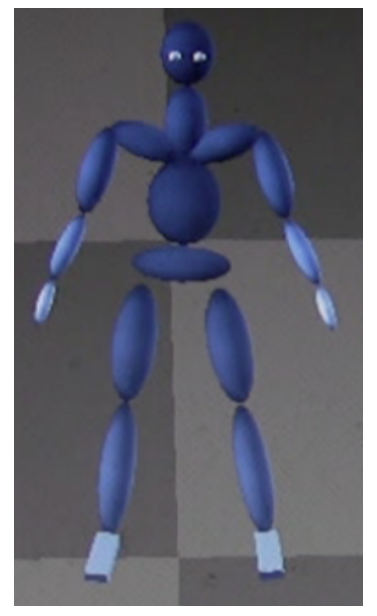

Fig. 3. Virtual human model used in Experiment 1.

In the experiment, videos of motions were used for data integrity and motion repeatability. For example, if the robot hardware were used in the motion capture lab, the infrared light would reflect off the aluminum robot body and corrupt data. The original retargeted and original coordinated motion trajectories were videotaped on the Simon hardware from multiple angles for the study. Similarly, the original human motion was visualized on a simplified virtual human character (Figure 3) and also recorded from multiple angles. Each video of the recorded motion contained all recorded angles shown serially. There were 60 input (i.e. stimulus) videos total (20 motions for the 3 groups described above).

Forty-one participants (17 women and 24 men), ranging in ages from 20-26 were recruited for the study. Each participant saw a set of twelve motions from the possible set of twenty that were randomly selected for each participant in such a way that each participant received four $\mathrm{OH}$, four OR, and four OC motions each. This provided a set of 492 mimicked motions total (i.e. 164 motions from each of three groups, with 8-9 mimicked examples for each of 20 motions).

a) Part One - Motion Capture Data Collection: Each participant was equipped with a motion capture suit and told to observe videos projected onto the wall in the motion capture lab. They were instructed to observe each motion as long as necessary (without moving) until they thought they could mimic it exactly. The video looped on the screen showing the motion from different view angles so participants could view each DOF with clarity. Unbeknownst to them, the number of views before mimicking (NVBM) was recorded as a measure for the study.

When the participant indicated they could mimic the motion exactly, the video was turned off and the motion capture equipment was turned on, and they performed one motion. Since there is a documented effect of practice on coordination [17], they were not allowed to move while watching and only their initial performance was captured. This process was repeated for the twelve motions. Prior to the twelve motions, each participant was allowed an initial motion for practice and to get familiar with the experimental procedure. Only when a participant was grossly off with respect to timing or some other anomaly occurred, were suggestions made about their performance before continuing. This happened with two participants, during their practice sessions, and those two participants' non-practice data is included in the experimental results. No practice data from any participant is included in the experimental results.

After mimicking each motion, the participant was asked if they recognized the motion, and if so, what name they would give it (e.g. wave, beckon). Participants did not select motion names from a list. After mimicking all twelve motions, the participant was told the original intent (i.e. name) for all 12 motions in their set. They were then asked to perform each motion unconstrained, as they would normally perform it. This data was recorded with the motion capture equipment, and in our analysis it is labeled the "participant unconstrained" (PU) set.

While the participants removed the motion capture suit, they were asked which motions were easiest and hardest to mimic; which motions were easiest and hardest to recognize; and which motion they thought that they had mimicked best (TMB). They were asked to give their reasoning behind all of these choices.

Thus, at the conclusion of part one of Experiment 1, the following data had been collected for each participant:

- Motion capture data from 12 mimicked motions:

- 4 "mimicking human" (MH) motions.

- 4 "mimicking retargeted" (MR) motions.

- 4 "mimicking coordinated" (MC) motions.

- Number of views before mimicking for each of the 12 motions above.

- Recognition (yes/no) for each of the 12 motions.

- For all recognizable motions, a name for that motion.

- Motion capture data from 12 "participant unconstrained" (PU) performances of the 12 motions above.

- Participant's selection of: 
- Easiest motion to mimic, and why.

- Hardest motion to mimic, and why.

- Easiest motion to recognize, and why.

- Hardest motion to recognize, and why.

- Which motion they thought that they mimicked the best, and why.

b) Part Two - Video Comparison: After finishing part one, participants watched pairs of videos for all twelve motions that they had just mimicked. Each participant watched the retargeted and coordinated versions (OR and OC) of the robot motion serially, but projected in different spatial locations on the screen to facilitate mental distinction. The order of the two versions was randomized. The videos were shown once each and the participants were asked if they perceived a difference. Single viewing was chosen because it leads to a stronger claim if difference can be noted after only one comparison viewing.

Then, the videos were allowed to loop serially and the participants were asked to watch the two videos and tell which motion in which video they thought looked "better" and which motion they thought looked more natural. The participants were also asked to give reasons for their choices. Unbeknownst to them, the number of views of each version before deciding "better" and more natural was also collected. Video order for all motions and motion pairs was randomized.

Thus, at the conclusion of part two of Experiment 1, the following data had been collected for each participant:

- Recognized a difference between retargeted and coordinated motion after one viewing (yes/no); for each of 12 motions mimicked in part one (Section VI-A.0.a)

- For motions where a difference was acknowledged,

- Selection of retargeted or coordinated as "better"

- Selection of retargeted or coordinated as more natural

- Rationale for "better" and more natural selections

- Number of views before each of these decisions

\section{B. Results}

1) H1: Human-like Optimization Increases Recognition:

The results presented in this section support Hypothesis 1, that our human-like optimization makes robot motion easier to recognize. The data in Fig. 4 represents the percentage of participants who named a motion correctly, incorrectly, or who opted not to try to identify the motion (i.e. unrecognized). This data is accumulated over all 20 motions and sorted according to the three categories of stimulus video: $\mathrm{OH}, \mathrm{OR}$, and OC. Coordinated robot motion was correctly recognized $87.2 \%$ of the time, and was mistakenly named only $9.1 \%$ of the time. These are better results than either human or retargeted motion. Additionally, coordinating motion led human observers to try to identify motions more frequently than human or retargeted motion (unrecognized $=3.7 \%$ for $\mathrm{OC}$, compared to $8.5 \%$ for $\mathrm{OH}$ and $11 \%$ for OR). This data suggests that the human-like optimization (i.e coordinating motion trajectories) makes the motion more familiar or common.

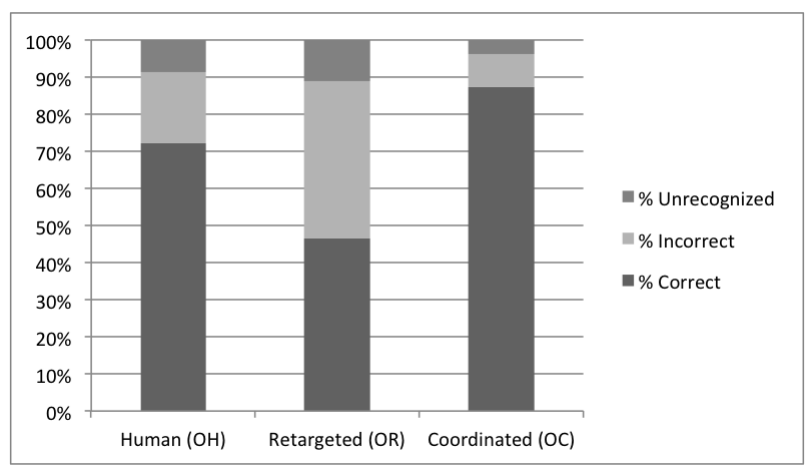

Fig. 4. Percent of motion recognized correctly, incorrectly, or not recognized by participants in Experiment 1, for each of the three categories of original data that they were asked to mimic. Spatio-temporal Coordinated motion is correctly recognized significantly more than simply retargeted motion, and at a rate similar to (but even higher than) the human motion.

On a motion-by-motion basis, percent correct was highest for 16 of 20 coordinated motions and lowest for 17 of 20 retargeted motions. In 17 of 20 motions percent incorrect was lowest for coordinated motions, and in a different set of 17 of 20 possible motions, percent incorrect was highest for retargeted motion. These numbers support the aggregate data presented in Fig. 4 suggesting that naming accuracy, in general, is higher for coordinated motion, and lower for retargeted motion. Comparing only coordinated and retargeted motion, percent correct was highest for 19 of 20 possible motions, and in a different set of 19 of 20 , percent incorrect was highest for retargeted motion. This data implies that relationships for recognition comparing retargeted and coordinated robot motion are maintained, in general, regardless of the particular motion performed. For reference, overall recognition of a particular motion (aggregate percentage) is a function of the motion performed. For example, waving was correctly recognized $91.7 \%$ of the all occurrences $(\mathrm{OH}, \mathrm{OR}$, and $\mathrm{OC})$, whereas 'flapping like a bird' was correctly recognized overall only $40.2 \%$ of the time.

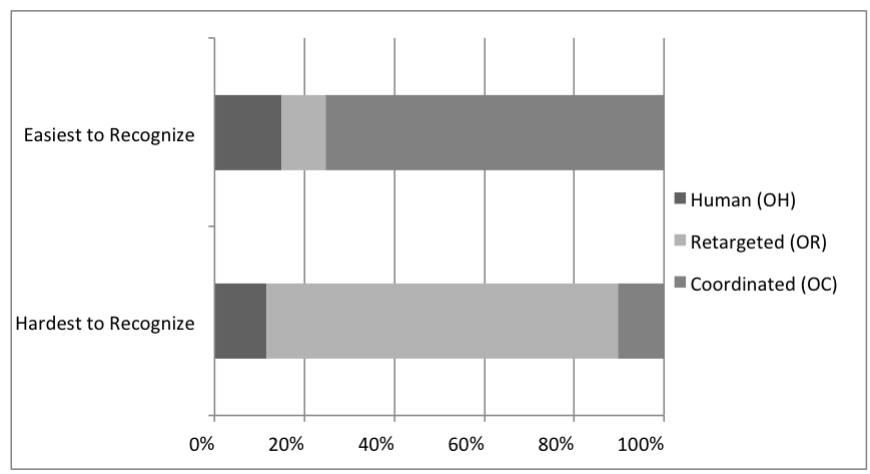

Fig. 5. Percent of responses selecting types of motions as easiest and hardest motion to recognize, for each of the three categories of original data that they were asked to mimic. Spatio-temporal Coordinated motion is most often selected as easiest, and Retargeted is most often selected as hardest.

The subjective data also supports the conclusion that 
coordinated motion is easier to recognize. When asked which of the 12 motions they mimicked was the easiest and hardest to recognize, coordinated was most often found easiest, and retargeted most often hardest. Figure 5 shows the percentage of participants that chose an $\mathrm{OH}, \mathrm{OR}$, or OC motion as easiest/hardest. $75.3 \%$ of participants chose a coordinated motion as the easiest motion to recognize, and only $10.2 \%$ chose a coordinated motion as the hardest motion to recognize. A significant majority of participants $(78.3 \%)$ selected a retargeted motion as the hardest motion to recognize.

When asked, participants claimed coordinated motion was easiest to recognize because it looked "better", "more natural", and was a "more complete" and "detailed" motion. And, retargeted motion was hardest because it looked "artificial" or "strange" to participants.

This notion of coordinated being "better" than retargeted is supported quantitatively by the second part of experiment 1 . In $98.98 \%$ of the trials, participants recognized a difference between retargeted and coordinated motion after only one viewing. When difference was noted, $56.1 \%$ claimed that coordinated motion looked more natural $(27.1 \%$ chose retargeted), and $57.9 \%$ said that coordinated motion looked "better" (compared with $25.3 \%$ for retargeted). In the remaining $16.8 \%$, participants (unsolicited) said that "better" or more natural depends on context, and therefore they abstained from making a selection. Participants who selected coordinated motion indicated they did so because it was a "more detailed" or "more complete" motion, closer to their "expectation" of human motion.

Statistical significance tests for the results in Figures 4 and 5 were not performed due to the nature of the data. Each number is an accumulation expressed as a percentage. The data is not forced choice; all participants were trying to correctly recognize the motion; some attempted and failed, and some did not attempt because they could not recognize the motion.

2) H2: SC and TC are better than STC: The following results from Experiment 1 support hypothesis $\mathrm{H} 2$, that optimizing based on composite STC rather than the individual components of SC and TC produces slightly worse results.

In Equation 3, the individual terms (spatial and temporal) on the right-hand side can be evaluated separately, rather than summing to form a composite STC. In our analysis, when the components were evaluated individually on a motion-bymotion basis, 20 of 20 retargeted motions exhibited statistical difference $(\mathrm{p}<0.05)$ from the human mimicked data and 0 of 20 coordinated motions exhibited correspondence that is not statistically different $(\mathrm{p}>0.05)$ than human data distribution. We will discuss these results in much more detail in Section VI-B.3.However, with the composite STC used as the metric, only 16 of 20 retargeted motions were statistically different than the original human performance $(p<0.05)$. Since the results were slightly less strong when combining the terms and using composite STC as the metric rather than analyzing SC and TC individually, we recommend that the SC and TC individual components be used independently as a metric for human-likeness.

3) H3: Makes Motion Human-like: Having completed discussion on the general hypotheses for our human-like optimization, we have not yet completely proven that the optimization improves human-likeness in the presence of different models, agents, or data loss. Our robot, discussed in Section III, represents a model or agent which is different kinematically and dynamically from a human. Thus, we can use Experiment 1 data to support our hypotheses regarding the effect of the human-like optimization on trajectories that are projected onto kinematic and dynamic hierarchies of degrees-of-freedom (e.g. agents, models) that are different. The difference can be the result of DOF correspondence issues, and projection causes trajectory data loss. We call such trajectories: imperfect, which in the instance of Experiment 1, means non-human-like.

The data from Experiment 1 presented in this section supports hypothesis $\mathrm{H} 3$, that the human-like optimization (i.e. spatiotemporally coordinating motion) makes robot motion more human-like. In subsequent sections, hypothesis $\mathrm{H} 3$ will be refined (via hypotheses H4 and H5, Experiments 2 and 3, respectively) to be more general. These subsequent sections will show that for any model or agent that does not perfectly match the agent (i.e. the original human from which the original trajectory was motion captured or developed) and for large quantities of data loss, the human-like optimization makes any motion trajectory closer to human-like. This will be true in our case because the original trajectories were captured from a human.

Four sets of motion-capture data exist from Experiment 1 part one (Section VI-A.0.a): mimicking human (MH), mimicking retargeted (MR), mimicking coordinated (MC), and participant unconstrained (PU) motion. Analysis must occur on a motion-by-motion basis. Thus, for each of the 20 motions, there is a distribution of data that captures how well participants mimicked each motion. For each participant, we calculated the spatial and temporal correspondence according to Equation 3, which resolved each motion into two numbers, one for each term on the right-hand side of the equation. For each motion, 8-9 participants mimicked OH, OR, and OC. Three times more data exists for the unconstrained version because regardless which constrained version a participant mimicked, they were still asked to perform the motion unconstrained. Thus for the analysis, we resolved MH, MR, MC, and PU into distributions for SC and TC across all participants. There are separate distributions for each of the 20 motions, yielding $4 \times 2 \times 20$ unique distributions. The goal was to analyze each of the SC and TC results independently on a motion-by-motion basis, in order to draw conclusions about MH, MR, MC, and PU. We used ANOVAs to test the following hypotheses:

- H3.1: Human motion is not independent of constraint. In other words, all the human motion capture data sets, (MH, MR, MC, and PU) do not have significantly different distributions. The $\mathrm{F}$ values, for all twenty motions, ranged from 7.2-10.8 (spatial) and 6.9-7.6 (temporal) which are greater than $F_{c r i t}=2.8$. Therefore, 
TABLE I

NUMBER OF MOTIONS WITH P $<0.05$ FOR PAIRWISE SPATIAL CORRESPONDENCE COMPARISON T-TESTS FOR THE INDICATED STUDY VARIABLES. NOTE: TABLE IS IDENTICAL FOR TEMPORAL CORRESPONDENCE.

\begin{tabular}{c|c|c|c|c|c|c|c|} 
& $\mathrm{OH}$ & $\mathrm{OR}$ & $\mathrm{OC}$ & $\mathrm{MH}$ & $\mathrm{MR}$ & $\mathrm{MC}$ & $\mathrm{PU}$ \\
\hline $\mathrm{OH}$ & $\mathrm{X}$ & 20 & 0 & 0 & 20 & 0 & 0 \\
\hline OR & $\mathrm{X}$ & $\mathrm{X}$ & 20 & 20 & 20 & 20 & 20 \\
\hline OC & $\mathrm{X}$ & $\mathrm{X}$ & $\mathrm{X}$ & 0 & 20 & 0 & 0 \\
\hline MH & $\mathrm{X}$ & $\mathrm{X}$ & $\mathrm{X}$ & $\mathrm{X}$ & 20 & 0 & 0 \\
\hline MR & $\mathrm{X}$ & $\mathrm{X}$ & $\mathrm{X}$ & $\mathrm{X}$ & $\mathrm{X}$ & 20 & 20 \\
\hline MC & $\mathrm{X}$ & $\mathrm{X}$ & $\mathrm{X}$ & $\mathrm{X}$ & $\mathrm{X}$ & $\mathrm{X}$ & 0 \\
\hline PU & $\mathrm{X}$ & $\mathrm{X}$ & $\mathrm{X}$ & $\mathrm{X}$ & $\mathrm{X}$ & $\mathrm{X}$ & $\mathrm{X}$ \\
\hline
\end{tabular}

we concluded at least one of these distributions is different from the others with respect to SC and TC.

- H3.2: Mimicked motion is not independent of constraint. In other words, all mimicked (i.e. constrained) data, (MH, MR, and MC) do not have significantly different distributions. In these ANOVA tests, values for all twenty motions ranged between 6.1-8.6 (spatial) and 5.3-6.6 (temporal), which are greater than $F_{\text {crit }}=$ 3.4-3.5. Therefore, we concluded that at least one of these distributions for mimicked motion is statistically different.

- H3.3: Coordinated motion is indistinguishable from human motion in terms of spatial and temporal coordination. $\mathrm{MH}, \mathrm{MC}$, and PU sets do not have significantly different distributions. $F_{\text {observed }}$ of 0.6-1.1 (spatial) and 0.9-1.9 (temporal), which are less than $F_{\text {crit }}$ of 3.23.3 , meaning that with this data there was insufficient evidence to reject this hypothesis for all twenty motions.

Since the above analysis isolated that retargeted motion was different from the other spatial and temporal correspondence distributions in mimicked motion, at this point, pairwise t-tests were performed to determine the difference between data sets on a motion-by-motion basis. Table I shows the number of motions for which there is a statically significant difference in spatial correspondence (the table for temporal correspondence is identical but not shown). For example, when participants mimicked retargeted motion, twenty motions were statistically different than the original retargeted performance. However, for the data when participants mimicked human or coordinated motion, the distributions failed to be different from their original performance for both spatial and temporal coordination (H3.3). From this, we conclude that humans are not able to mimic retargeted motion as well as the coordinated or human motion.

Since the above statistical tests do not allow us to conclude that the distributions were identical (H3.3), we performed a regression analysis of the data across all twenty motions to determine how correlated any two variables are in the study. For the purpose of this regression analysis the variables are either the mean or the standard deviation of SC, TC, or STC, for each of the distributions $(\mathrm{OH}, \mathrm{OR}, \mathrm{OC}, \mathrm{MH}$, $\mathrm{MR}, \mathrm{MC}, \mathrm{PU})$. However, the original data sets $(\mathrm{OH}, \mathrm{OR}$,
TABLE II

$R^{2}$ VALUE FROM LINEAR REGRESSION ANALYSIS ON SPATIAL (SC), TEMPORAL (TC) AND COMPOSITE MEAN CORRESPONDENCE (STC) FOR PAIRS OF VARIABLES. $R^{2}=1$ (PERFECTLY CORRELATED); $0=$ (UNCORRELATED). NOTE THE HIGH CORRELATION BETWEEN MIMICKED HUMAN AND COORDINATED MOTIONS SEEN IN ROW 14.

\begin{tabular}{l|l|c|c|c|} 
& Variables & SC & TC & STC \\
\hline 1 & OH v. MH & 0.9783 & 0.9756 & 0.9914 \\
\hline 2 & OH v. MR & 0.6339 & 0.0427 & 0.5483 \\
\hline 3 & OH v. MC & 0.9792 & 0.965 & 0.9933 \\
\hline 4 & OH v. PU & 0.9859 & 0.9378 & 0.9843 \\
\hline 5 & OR v. MH & 0.0103 & 0.0009 & 0.0022 \\
\hline 6 & OR v. MR & 0.0915 & 0.008 & 0.0526 \\
\hline 7 & OR v. MC & 0.0001 & 0.0002 & 0.0004 \\
\hline 8 & OR v. PU & 0.0011 & 0.0003 & 0.0001 \\
\hline 9 & OC v. MH & 0.9494 & 0.9626 & 0.9819 \\
\hline 10 & OC v. MR & 0.6084 & 0.0491 & 0.5176 \\
\hline 11 & OC v. MC & 0.9834 & 0.962 & 0.9918 \\
\hline 12 & OC v. PU & 0.9836 & 0.9414 & 0.9795 \\
\hline 13 & MH v. MR & 0.6412 & 0.0421 & 0.5612 \\
\hline 14 & MH v. MC & 0.9531 & 0.9749 & 0.9809 \\
\hline 15 & MH v. PU & 0.969 & 0.9271 & 0.9756 \\
\hline 16 & MR v. MC & 0.6728 & 0.0516 & 0.5365 \\
\hline 17 & MR v. PU & 0.6414 & 0.017 & 0.5076 \\
\hline 18 & MC v. PU & 0.9881 & 0.9144 & 0.9822 \\
\hline
\end{tabular}

TABLE III

$R^{2}$ VALUE FROM LINEAR REGRESSION ANALYSIS ON STANDARD DEVIATION OF SPATIAL, TEMPORAL AND COMPOSITE CORRESPONDENCE FOR PAIRS OF STUDY VARIABLES. $R^{2}=1$ (PERFECTLY CORRELATED); 0

$=($ UNCORRELATED $)$. VARIABLES NOT SHOWN HAVE A STANDARD DEVIATION OF 0. NOTE THE HIGH CORRELATION BETWEEN MIMICKED HUMAN AND COORDINATED MOTIONS SEEN IN ROW 2.

\begin{tabular}{c|l|c|c|c|} 
& Variables & SC & TC & STC \\
\hline 1 & MH v. MR & 0.1005 & 0.1231 & 0.3507 \\
\hline 2 & MH v. MC & 0.8847 & 0.7435 & 0.9842 \\
\hline 3 & MH v. PU & 0.0674 & 0.0906 & 0.8348 \\
\hline 4 & MR v. MC & 0.0746 & 0.1749 & 0.346 \\
\hline 5 & MR v. PU & 0.5002 & 0.0002 & 0.2239 \\
\hline 6 & MC v. PU & 0.0986 & 0.096 & 0.8537 \\
\hline
\end{tabular}

and OC) are only one number (not a distribution) so they were not included in the standard deviation analysis. The intuition for this analysis is that if two variables are highly correlated with respect to both mean and variance, then it is further evidence that their distributions are similar. Specifically, results showing high correlation between the human and coordinated motions were expected, if Hypothesis 3 is to be supported.

The $R^{2}$ values from the linear data fits, are shown in Tables II and III. This data shows that participants mimicking coordinated and human motion were highly correlated (line 14 in Table II and line 2 in Table III, lightly shaded), whereas the data from when participants mimicked retargeted motion was less correlated to all other data including the original human performance (lines 2, 6, 10, 13, and 16 in Table II). When two variables have high correlation in a linear data fit, it means that either variable would be a excellent linear predictor of the other variable in the pair. These 
higher correlations between human and coordinated motion are further evidence that coordinated motion is more humanlike than retargeted motion.

Furthermore, the standard deviation correlation on line 3 in Table III is low for the spatial and temporal components when regressing mimicked human and participant unconstrained data, which shows that mimicking does in fact constrain people's motion. Variance increases for the PU distribution because motion is unconstrained and humans are free to perform the motion as they please. This validates our premise in Experiment 1 that mimicking performance is a better method by which to compare motion.

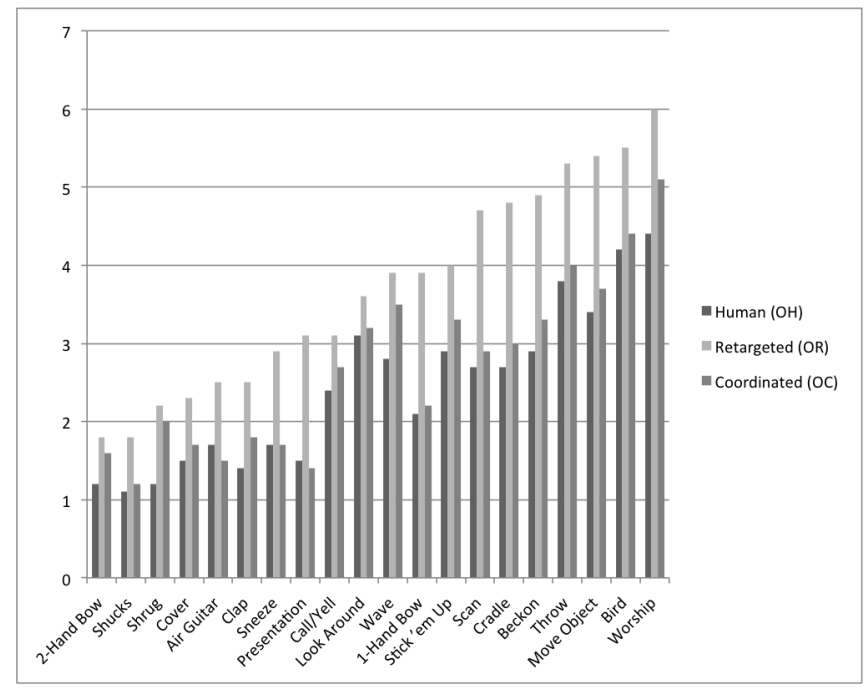

Fig. 6. Average number of times participants watched each motion before deciding that they were prepared to mimic the motion. Retargeted versions of the motion always required the most views, whereas coordinated and human motion were usually more similar in number of views before mimicking.

The data in Figure 6 shows number of views before mimicking for each of the 20 motion, which also supports the claim that coordinated motion is more human-like. On average, humans viewed a retargeted motion more times before they are able to mimic (3.7 times) as compared to coordinated motion (2.7 times) or human motion (2.4 times). Pairwise t-tests between these distributions, on a motion-bymotion basis for NVBM, showed that 19 of 20 retargeted motions exhibited statistical significance $(p<0.05)$ when compared with human NVBM whereas only 3 of 20 coordinated motions NVBM were statically different $(\mathrm{p}<0.05)$ from human NVBM. This suggests coordinated motion is more similar to human motion in terms of preparation for mimicking.

Of the 12 mimicked motions, each participant was asked which motion was easiest and hardest to mimic. Of all participant responses, $75.6 \%$ of motions chosen as easiest were coordinated motions, and only $12.2 \%$ of participant responses chose a coordinated motion as hardest to mimic (Fig. 7). In the assertion stated earlier, we claimed that a human would be able to more easily mimic something common and familiar to them. These results suggest that coordination

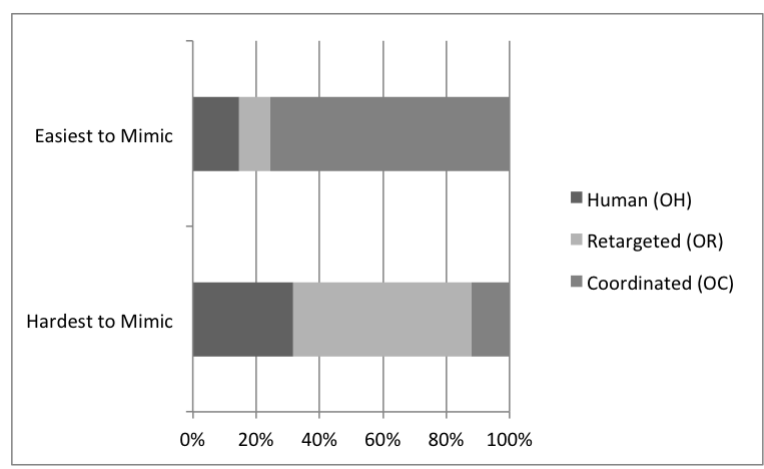

Fig. 7. Percent of responses selecting types of motions as easiest and hardest motion to mimic. Coordinated is usually selected as easiest, and retargeted as hardest.

adds this quality to robot motion, which improves not only ability to mimic, as presented earlier, but also perception of difficulty in mimicking (Fig. 7).

During questioning of participants in post-experiment interviews, we gained insight into people's choices of easier and harder to mimic. Participants felt that human and coordinated motion were "more natural" or "more comfortable." Participants also indicated that human and coordinated motion were easier to mimic because the motion was "more familiar," "more common," and "more distinctive." In comparison, some people selected retargeted motion as being easier to mimic because fewer parts are moving in the motion. Others said retargeted motion is hardest to mimic because the motion felt "artificial" and "more unnatural."

\section{Summary of Experiment 1}

The purpose of experiment 1 was to support the general hypothesis that increasing spatio-temporal correspondence of distributed actuators synthesizes more human-like motion. Our experimental design asked people to mimic motion that was either human motion, human motion retargeted to a humanoid robot, or additionally spatio-temporal coordinated motion. We find that people can more accurately recognize or infer the intent of STC motion, and they subjectively report this to be so. We show that SC and TC are more accurate metrics when used separately than in combination. Then find that based on such a metric, find that people are better able to mimic exactly a human motion or a coordinated motion, and are significantly worse at accurately mimicking retargeted motion. And this is also their subjective experience, most people found retargeted motion to be the hardest to mimic.

\section{EXPERIMENT 2: HUMAN MOTION}

The purpose of Experiment 2 is to further quantitatively support that spatio-temporal correspondence of distributed actuators is a good metric for human-like motion. Since human motion exhibits spatial and temporal correspondence, if SC and TC are good metrics for human motion, then optimizing human motion with respect to these metrics should not significantly change the spatial and temporal correspondence of human motion. 


\section{A. Experimental Design}

Experiment 2 was designed to test hypothesis H4, which is an extension to hypothesis H3. In Experiment 1, we tested retargeting human motion onto two different models: a human model and the Simon robot model. By doing so, we demonstrated that the human-like optimization recovers information lost when the model is imperfect. To state it precisely, an imperfect model is any model that is kinematically or dynamically different than the original agent who was motion captured. In Experiment 1, statistical difference of SC and TC groups for the retargeted robot motions showed that the retargeting process loses data when projecting onto an imperfect model (i.e., retargeting the human motion to the robot model). The advantage of the human-like optimization was that it was able to recover this lost data (e.g., we saw high correlation of the coordinated motion and human motion).

In Experiment 2, we focus on the human data to add evidence that SC and TC are good metrics for human motion. Hypothesis $\mathrm{H} 4$ extends $\mathrm{H} 3$ by demonstrating a complementary scenario: that less motion data is lost when models that are closer to ideal are used (i.e. the human-like optimization is unnecessary for projection onto ideal models).

We did not explain the details in Experiment 1, but the human model used for the motion captured datasets (i.e. the $\mathrm{OH}, \mathrm{MH}$, and PU) was actually 42 different human models (i.e. one for each participant and one for the human who performed the original, initial motions). The optimization for SC and TC in Equation 3 uses torque trajectories, and each human participant in Experiment 1 was physically different (e.g. mass, height, strength). In order to get accurate dynamic models for our human data, when Experiment 1 was performed, we collected basic anthropometric data from our participants: height, weight, gender, and age. Using the model scaling functionality of the Software for Interactive Musculoskeletal Modeling (SIMM) Tool ${ }^{1}$ and the dynamic parameters we collected, we were able to produce accurate dynamic models of all humans, from which we collected motion capture data.

In Experiment 1, human participants were visualizing the $\mathrm{OH}$ data on the simplified human model shown in Figure 3. Although the SIMM tool provides trajectories for 86 DOFs, in the motion capture data, markers were not placed to sufficiently capture all 86 DOFs because we focus on body motion in this research. Thus, the number of DOFs in the human model was also simplified. Degrees-of-freedom in the eyes, thumbs, toes, ears, and face of the human model were removed since motion capture markers were not placed to capture sufficient motion in these areas when we collected data. Additional DOFs were removed in the human model in locations such as the legs, since the majority of these degreesof-freedom were not significantly animated in the 20 motions from Experiment 1 (i.e. they are upper body motions). The final human model was comprised of 34 joints, concentrated in the neck, abdomen, and arms. When the motion capture

\footnotetext{
${ }^{1}$ Trademark of MusculoGraphics Inc. All rights reserved.
}

data was retargeted to the human model, 45 markers were used as constraints to animate the 34 DOFs.

After each model was scaled for dynamic parameters unique to each participant (thereby creating 42 human models), the human motion capture data was retargeted to these 34-DOF simplified human models optimally. From this optimal retargeting, we had the original human and mimicked human $(\mathrm{OH}$ and $\mathrm{MH})$ data sets. Since each participant in Experiment 1 mimicked 4 original human motions, we had distributions of mimicked human data formed from 89 human performances per motion. For Experiment 2, we combined these $\mathrm{OH}$ and $\mathrm{MH}$ data sets to create a data set that we call pre-optimization (pre-op), which consists of 9-10 similar human examples for each of 20 different motions.

To test H4, we optimized each of these 184 trajectories (4 MH motions per participant x 41 participants $+20 \mathrm{OH}$ motions) according to the procedure in Section IV-A. This provided a comparison dataset of 9-10 similar human examples for each of 20 different motions optimized according to the STC metric. This set is called post-optimization (postop).

The SC and TC were evaluated for each of the trajectories in the pre-op and post-op data sets to create paired distributions of SC and TC for each motion. According to hypothesis $\mathrm{H} 4$, if SC and TC are good metrics for human-like motion, the optimization should not significantly affect them when the models used for retargeting and optimization are close to identical.

\section{B. Results}

For the initial analysis, on a motion-by-motion basis, twenty pre-op and twenty post-op distributions were combined to create twenty distributions (one for each motion). H4 states that SC and TC will not be affected by the humanlike optimization when the data is not modified and the model accurately represents the agent who was motion captured. In other words, the SC and TC of the pre-op and post-op data come from the same distribution.

Twenty F-tests were performed, i.e. one for each combined motion distribution. The $\mathrm{F}$ values, for all twenty motions, ranged from 0.7-1.3 (spatial) and 0.8-1.4 (temporal) which were less than $F_{c r i t}=4.4-4.5$. Therefore, the data for all 20 motions was not statistically different before and after the optimization with respect to SC and TC. This data does not allow us to prove $\mathrm{H} 4$ definitively, but it is a first step toward understanding the performance of our human-like optimization. Since we believe that the metric is valid, we can increase confidence that the pre-op and post-op data belong to the same distribution with a regression analysis.

Assuming a normal distribution, high correlation between the pre-op and post-op distributions' mean values and standard deviations would increase confidence in H4. Using the twenty independent data points (one for each motion), we formed linear regressions for the pre-op and post-op distributions' mean values and standard deviations. After performing these four linear regressions, the correlation coefficient of the means was 0.9874 (SC) and 0.9657 (TC), and the standard 
deviations resulted in a correlation coefficient of 0.9791 (SC) and 0.9580 (TC). Ideally, the correlation coefficient of both of these regressions would be 1.0 , which would indicate that they are identical distributions. The corresponding slopes of these two lines were 0.9954 (mean, SC), 0.9879 (mean, TC), 0.9481 (standard deviation, SC), and 0.9823 (standard deviation, TC). The ideal slope is 1.0, and these results show that the optimization did not significantly affect SC or TC of human-like motion when the models used for retargeting and optimization were identical.

\section{Summary of Experiment 2}

To summarize, in experiment 2 we have strengthened the proof that spatio-temporal correspondence is a good metric for human-like motion. In particular we have shown that when the optimization is applied to motion that is already human-like (e.g., the $\mathrm{OH}$ and $\mathrm{MH}$ datasets from experiment $1)$, the resulting motion is not significantly different from the original. Moreover, the SC and TC measures are highly correlated for both the pre-optimized and post-optimized versions of these motions.

In experiment 1 we learned that the human-like optimization procedure described in Sec. IV-A can make retargeted motion more natural looking and easier for people to identify intent. Experiment 2 has additionally shown that applying our optimization to motion that is already human-like does not alter it significantly; that the human-likeness is left in tact.

\section{EXPERIMENT 3: Downsampling Human Motion}

This next experiment is designed to test $\mathrm{H} 5$, that our human-like motion optimization can work to recover information loss in situations with imperfect data.

In the last experiment, we provided evidence that the optimization does not significantly affect SC and TC for human-like motion when the model is identical for retargeting and optimization. With Experiment 3, we want to test the strength of the optimization in "retrieving" lost information and data. In other words, after Experiment 2 we knew that the optimization would function as expected under ideal conditions (i.e. ideal model and ideal data), but in Experiment 3 we wanted to test the metric to see whether it would function as expected when used in nonideal conditions. One such non-ideal condition was discussed in Experiment 1: when the motion trajectory is used on an agent that was kinematically and dynamically different than a human (i.e. a robot). Experiment 3 tests the remaining nonideal scenario.

$K_{2}$ in Equation 3 from the human-like optimization is a metric from chaos theory that estimates the information lost as a function of time for a stochastic signal. In the humanlike optimization, we used KSE to estimate information difference between two deterministic signals (i.e. proximal DOF trajectories) and by correlating these two signals the robot motion became more human-like. Thus, in Experiment 3 , we intentionally eliminate some information in the motion signal for an optimal model by downsampling the motion signal, and then we test how much of that information the optimization process is able to return for human motion. For example, this information loss could be due to motion trajectory transmission across a noisy channel or recording motion capture data at too low of a frequency.

\section{A. Experimental Design}

For Experiment 3, we use the same human model and same two data sets from Experiment 2 (pre-op and postop) for all 20 motions. We created eight new datasets, each of which represents the pre-op data set uniformly subsampled to remove information from the 184 trajectories. There were eight new data sets because the subsampling occurs at eight distinct rates: downsampling by rates in half integer intervals ${ }^{2}$ from 1.5 to 5.0 (i.e. 1.5, 2.0,..,5.0). These data sets are denoted by the label "pre-op" followed by the sampling rate (e.g. pre-op1.5 refers to the pre-op data set of trajectories with each trajectory subsampled by a rate of 1.5). For reference, the original pre-op data set from Experiment 2 represents pre-op1.0.

Then, we optimized each of the 1,472 trajectories (184 $x$ 8) according to the procedure in Section IV-A to create an additional eight new datasets for each of 20 motions. This provided paired comparison datasets of 9-10 similar human examples of each of 20 different motions for each of 8 unique sampling rates optimized according to the SC and TC metrics. These eight data sets are denoted by the label "postop" followed by the sampling rate (e.g. post-op1.5 refers to the pre-op1.5 dataset after optimization).

For each trajectory in these 16 new datasets $(2,944$ trajectories), SC and TC were evaluated. According to hypothesis $\mathrm{H} 5$, if SC and TC are good metrics for human-like motion, the optimization should compensate for SC and TC lost in the downsampling process when the models used for retargeting and optimization are identical. Also, H5 states that SC and TC for post-opN trajectories with subsampling rates closer to 5.0 will be less similar to the pre-op1.0 and post-op1.0 datasets for each motion.

\section{B. Results}

Since there are a large number of variables in the statistical significance tests (which result in many combinations of statistical tests), we will omit the details of the intermediate series of numerous tests that begin from the most broad hypothesis (i.e. that sampling rate has no effect on the pre-op1.0 trajectories; this means the optimized, downsampled, and original data (pre-op1.0, post-op1.0, pre-op1.5, post-op1.5,..., pre-op5.0, post-op5.0) come from the same distribution). The intermediate set of tests led us to conclude the following:

1) Downsampled trajectories prior to optimization (pre$\mathrm{opN})$ were significantly different $(\mathrm{p}<0.05)$ from preop1.0 and post-op1.0 for all sample rates $\mathrm{N}>1.0$, on a motion by motion basis for all 20 motions (with respect to both SC and TC). Thus we conclude that

\footnotetext{
${ }^{2}$ For non-integer subsampling, the rates are represented as a rational fraction $\mathrm{L}$ over $\mathrm{M}$ (e.g. $1.5=3 / 2 ; \mathrm{L}=3 ; \mathrm{M}=2$ ). The data is upsampled by linear interpolation by integer $\mathrm{L}$ first, followed by downsampling by integer M.
} 
degradation does cause a motion trajectory to lose spatial and temporal information.

2) Downsampled trajectories prior to optimization (pre$\mathrm{opN})$ were significantly different $(\mathrm{p}<0.05)$ with respect to downsampled trajectories after optimization (post$\mathrm{opN}$ ) for all evaluated sample rates $\mathrm{N}>1.0$, on a motion by motion basis for all 20 motions (with respect to both SC and TC). We conclude that the humanlike optimization significantly changed the spatial and temporal information of downsampled trajectories.

3) Downsampled trajectories prior to optimization (pre$\mathrm{opX})$ were significantly different $(\mathrm{p}<0.05)$ from each other (pre-opY) on a motion by motion basis for all 20 motions (with respect to both SC and TC) for all sample rates where $\mathrm{X} \neq \mathrm{Y}$. We conclude that downsampling at higher rates caused more spatial and temporal motion information to be lost.

The remainder of the pairwise t-tests are captured in Figure 8. In Fig. 8 the data from Experiment 2 (i.e. pre-op1.0 and post-op1.0) is combined into a single distribution and compared against the SC and TC data from downsampled datasets after optimization (i.e. post-opN, where $\mathrm{N}>1.0$ ).

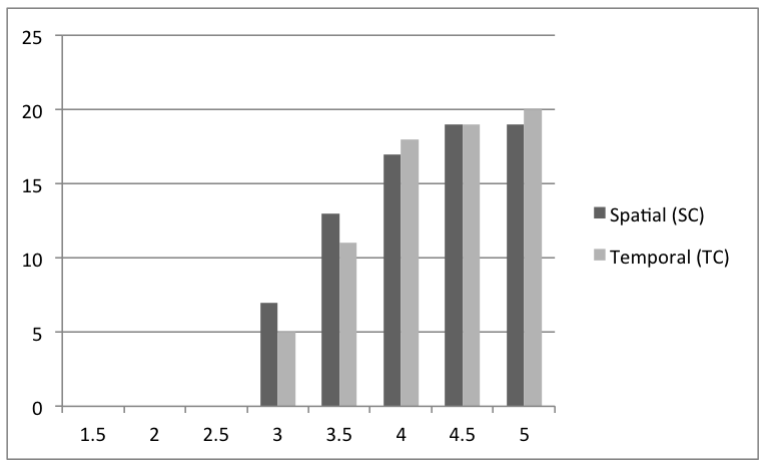

Fig. 8. Number of motions with $\mathrm{p}<0.05$ for pairwise spatial and temporal correspondence comparison t-tests for the composite data set of pre-op \& post-op (from Experiment 2) vs. post-opN, where $\mathrm{N}=$ downsample rate. For downsample rates less than 4.0 the motion is often not significantly different than the non-downsampled version.

Numbers closer to 20 in Fig. 8 indicate that more motions were unable to be recovered and restored with respect to SC or TC, as compared to the respective distributions without downsampling. Too much information was lost at higher sample rates $(N \geq 4.0)$, and the optimization was not able to compensate for the lost data, which provides evidence for the second half of H5. For lower values of downsampling rates, the optimization was able to perform well and recover lost information, but as downsampling rate increased less information was recoverable.

We would further like to say that for the sample rates $N<4.0$ the resulting optimized motion is similar to the pre-op1.0 and post-op1.0 distributions. To do so we perform a linear regressions on the distribution means and standard deviations (pre-op1.0 \& post-op1.0 vs. post-opN). The slopes and correlation coefficients, as shown in Tables IV and $\mathrm{V}$, indicate that for motion distributions that were
TABLE IV

SLOPES AND CORRELATION COEFFICIENTS $\left(R^{2}\right)$ OF MEAN $(\mu)$ AND STANDARD DEVIATION $(\sigma)$ FOR LINEAR REGRESSIONS OF SC OF PRE-OP $\&$ POST-OP (FROM EXPERIMENT 2) VS. POST-OPN, WHERE N = DOWNSAMPLE RATE. ONLY DATA FROM NON-STATISTICALLY SIGNIFICANT MOTIONS IS INCLUDED IN REGRESSIONS. SAMPLE RATES NOT SHOWN DID NOT INCLUDE ENOUGH DATA TO REGRESS A NON-TRIVIAL LINE.

\begin{tabular}{c|c|c|c|c|} 
& \multicolumn{4}{|c}{ Spatial, SC } \\
\hline $\mathrm{N}$ & $\mu$, Slope & $\mu, R^{2}$ & $\sigma$, Slope & $\sigma, R^{2}$ \\
\hline \hline 1.5 & 0.983 & 0.995 & 0.942 & 0.954 \\
\hline 2.0 & 0.976 & 0.957 & 0.941 & 0.958 \\
\hline 2.5 & 0.945 & 0.965 & 0.972 & 0.970 \\
\hline 3.0 & 0.967 & 0.955 & 0.953 & 0.975 \\
\hline 3.5 & 0.950 & 0.961 & 0.996 & 0.957 \\
\hline
\end{tabular}

TABLE V

SLOPES AND CORRELATION COEFFICIENTS $\left(R^{2}\right)$ OF MEAN $(\mu)$ AND STANDARD DEVIATION $(\sigma)$ FOR LINEAR REGRESSIONS OF TC OF PRE-OP $\&$ POST-OP (FROM EXPERIMENT 2) VS. POST-OPN, WHERE N = DOWNSAMPLE RATE. ONLY DATA FROM NON-STATISTICALLY SIGNIFICANT MOTIONS IS INCLUDED IN REGRESSIONS. SAMPLE RATES NOT SHOWN DID NOT INCLUDE ENOUGH DATA TO REGRESS A NON-TRIVIAL LINE.

\begin{tabular}{c|c|c|c|c|} 
& \multicolumn{4}{|c}{ Temporal, TC } \\
\hline $\mathrm{N}$ & $\mu$, Slope & $\mu, R^{2}$ & $\sigma$, Slope & $\sigma, R^{2}$ \\
\hline \hline 1.5 & 0.969 & 0.975 & 0.980 & 0.971 \\
\hline 2.0 & 0.951 & 0.942 & 0.947 & 0.943 \\
\hline 2.5 & 0.995 & 0.947 & 0.994 & 0.951 \\
\hline 3.0 & 0.982 & 0.967 & 0.969 & 0.973 \\
\hline 3.5 & 0.963 & 0.972 & 0.987 & 0.972 \\
\hline
\end{tabular}

not statistically different, the pre-op 1.0 , post-op 1.0 , and postopN distributions are similar (slopes close to 1.0 with high correlation coefficient). This means that for downsampled trajectories where sufficient information content survives the downsampling process, after optimization, the distributions of SC and TC of these trajectories (on a motion by motion basis) appeared similar to the distributions where there is no information loss (pre-op and post-op from Experiment 2, without downsampling data).

\section{Summary of Experiment 3}

Experiment 3 is our final piece of evidence supporting that the human-like optimization that we have proposed is a good one. In experiment 1 we showed it makes non-humanlike motion more human-like. In experiment 2 we showed that is doesn't degrade already human-like motion. And in experiment 3 , we intentionally eliminate some information in the motion signal (by downsampling), and show that the optimization process is still able to recover that information to produce a human-like motion.

\section{EXPERIMENT 4: HUMAN-LIKE VARIANTS}

Experiments 1, 2, and 3 showed that human trajectories projected onto a robot kinematic hierarchy are more human- 
like if they are optimized with respect to spatiotemporal correspondence. Now we would like create human-like variants for robot motion, which is why our variance optimization follows serially after the human-like optimization. For experiment 4 we analyze the human-likeness after this variance optimization.

\section{A. Experimental Design}

To demonstrate that the variance optimization maintains human-likeness when the optimized motion is already human-like, Experiment 4 proceeded in four-steps: (1) In Experiments 1-3, we already supported that the input to the variance algorithm is human-like motion. (2) After the variance optimization, the SC and TC of the variants should not show statistical difference from the human-like optimization input motion SC and TC respective values. (3) Since step two does not allow us to make any claims about distribution equality between input and output motions for the variance optimization, we show high correlation between these distributions using a regression analysis. (4) Finally, to strengthen our quantitative data from step three we performed an experiment that is explained in the next section.

For step four, the twenty motions from Experiment 1 that represent the original coordinated data set were used as inputs to the variance optimization. These are the SC and TC coordinated versions of the human motion executed on the robot hardware. The first twelve variants of each motion were generated, according to the procedure described in Sec. IV-B, and videos were made of these variants running on the robot hardware. Three different viewing angles were used to help participants see the motions clearly. These variants are labeled V1 to V12, and V0 represents the input motion to the variance optimization. Videos were used so that participants would be able to rewatch motions more easily.

Order of the twenty motions was randomized for each participant. The thirteen videos (one input and 12 variants) for the first randomly selected motion were shown to the participant in a random order. The participant was asked to select the most human-like motion from all thirteen. Participants were allowed to rewatch any videos in any order as many times as desired before making their selection. The experiment concluded when the most human-like version for all twenty motions was selected.

\section{B. Results}

The SC and TC of the variance optimization outputs (i.e, the motion variants) should not show statistical difference from the human-like motions' (i.e. variance optimization inputs) SC and TC respective values. Thus, we compare the SC and TC distributions before and after the variance optimization. The original coordinated data set is only one data point per motion prior to the variance optimization (i.e. the variance optimization input, which is the original coordinated data from Experiment 1, is not a distribution for each motion), and neither SC nor TC can be averaged across motions. We are making comparisons of human-likeness on the robot hardware, and therefore we have a couple options for comparison data sets from Experiment 1 that will produce distributions that are representative for motions after the human-like optimization. The following are valid representative distributions after the human-like optimization because the analysis in Experiment 1 showed high correlation between these distributions (i.e. they were representative of human-like motion on the robot hardware).

- OC (Original Coordinated): This is the original human $(\mathrm{OH})$ data set from Experiment 1, optimally retargeted to the robot model (which became the original retargeted data set), and then processed through the human-like optimization. This is identical to the original coordinated data set from Experiment 1. 1 example per motion.

- MHC (Mimicked Human Coordinated): This is the mimicked human $(\mathrm{MH})$ data set from Experiment 1, optimally retargeted to the robot model, and then processed through the human-like optimization. 8-9 examples per motion.

- MCC (Mimicked Coordinated Coordinated): This is the mimicked coordinated (MC) data set from Experiment 1 , optimally retargeted to the robot model, and then processed through the human-like optimization. 8-9 examples per motion.

- PUC (Participant Unconstrained Coordinated): This is the participant unconstrained (PU) data set from Experiment 1 , optimally retargeted to the robot model, and then processed through the human-like optimization. 2427 examples per motion.

The latter three options (MHC, MCC, and PUC) represent different sets of human participant data from Experiment 1 (i.e. the first two acronym characters), which undergo optimal retargeting and then the human-like optimization. Each of the four options (after the human-like optimization but before the variance optimization) had twelve variants generated per example per motion, to create a respective comparison data set distribution after the variance optimization.

We performed pairwise t-tests on each of these four datasets (OC, MHC, MCC, and PUC) compared against their respective data set after the variance optimization, and found that none of these tests showed statistical difference in the SC or TC dimension. This begins to support H6, that the variance algorithm does not alter human-likeness of the input motion. However, a regression analysis is required to provide conclusive support.

Tables VI and VII show that the distribution of spatial and temporal correspondence is fairly well maintained before and after variance optimization, regardless of the human-like motion data on the robot that is used. The high correlation numbers and slopes close to one provide insight that under the assumption of Normal distributions for this data, the variance optimization does not significantly change the distribution. These distributions represent the amount of spatial and temporal coordination in the motions, and it is important to remember that SC and TC are not measures of amount of variance in motion. 
TABLE VI

$R^{2}$ AND SLOPE VALUES FROM LINEAR REGRESSION ANALYSIS ON MEAN OF SPATIAL AND TEMPORAL CORRESPONDENCE FOR PAIRS OF STUDY VARIABLES (BEFORE VS. AFTER THE VARIANCE OPTIMIZATION). $R^{2}=1$ (PERFECTLY CORRELATED); $0=($ UNCORRELATED $)$. SLOPE $=1$ IS IDEAL. LINE REGRESSED FROM 20 MOTION DATA POINTS.

\begin{tabular}{c|l|c|c|c|c|}
\multicolumn{2}{c|}{} & \multicolumn{2}{|c|}{ Spatial, SC } & \multicolumn{2}{c|}{ Temporal, TC } \\
& Set & Slope & $R^{2}$ & Slope & $R^{2}$ \\
\hline 1 & OC & 0.8640 & 0.8824 & 0.8003 & 0.8231 \\
\hline 2 & MHC & 0.8434 & 0.9602 & 0.8115 & 0.9395 \\
\hline 3 & MCC & 0.9089 & 0.8668 & 0.8549 & 0.9176 \\
\hline 4 & PUC & 0.8907 & 0.9421 & 0.8744 & 0.9299 \\
\hline
\end{tabular}

TABLE VII

$R^{2}$ AND SLOPE VALUES FROM LINEAR REGRESSION ANALYSIS ON STANDARD DEVIATION OF SPATIAL AND TEMPORAL CORRESPONDENCE FOR PAIRS OF STUDY VARIABLES (BEFORE VS. AFTER THE VARIANCE OPTIMIZATION). $R^{2}=1$ (PERFECTLY CORRELATED); $0=$ (UNCORRELATED). SLOPE $=1$ IS IDEAL. LINE REGRESSED FROM 20

MOTION DATA POINTS. OC IS NOT A DISTRIBUTION AFTER THE HUMAN-LIKE OPTIMIZATION (I.E. NO STANDARD DEVIATION) AND IS NOT SHOWN.

\begin{tabular}{c|l|c|c|c|c|}
\multicolumn{2}{c|}{} & \multicolumn{2}{|c|}{ Spatial, SC } & \multicolumn{2}{c|}{ Temporal, TC } \\
& Set & Slope & $R^{2}$ & Slope & $R^{2}$ \\
\hline 1 & MHC & 0.9036 & 0.9825 & 0.9213 & 0.9642 \\
\hline 2 & MCC & 0.8771 & 0.9469 & 0.9326 & 0.9221 \\
\hline 3 & PUC & 0.9247 & 0.8841 & 0.8934 & 0.8793 \\
\hline
\end{tabular}

To strengthen our evidence that the variance optimization does not significantly affect human-likeness in motion data, we supplement our quantitative data with qualitative data. For step four (i.e. the experiment involving participants) only the original coordinated version after the human-like optimization was used because using additional data would result in the requirement of too many participants for all experimental conditions.

100 participants were recruited on the Georgia Institute of Technology campus (57 male, 43 female; ranging in age from 19-40). 100 participants yields a data resolution of $1 \%$ accuracy for results expressed as percentages.

In prior work, we established that torque space distance is a good indicator of degree of difference between two static poses (i.e. time instant states) in a motion [9]. We now order the results from this experiment for all twelve variants according to increasing torque space distance from the input motion (i.e. the original coordinated version was used to create the variants) for all 20 motions. For this particular experiment, the torque-space distance metric allows us to order the variants by increasing variance from the original motion that created them.

Table VIII shows that when the first 12 variants are ordered by increasing torque space distance from input motion (i.e. the original coordinated motion), the percent of participants choosing each variant as the most human-like of all 13 versions for a given motion is fairly uniform. Recall, that
TABLE VIII

PERCENT OF PARTICIPANTS WHO SELECTED A VARIANT AS THE MOST HUMAN-LIKE FROM THE SET OF OC AND THE FIRST 12 VARIANTS GENERATED B Y THE VARIANCE OPTIMIZATION. ALL DATA IN THE TABLE ARE PERCENTAGES. THE VARIANTS ARE ORDERED BY INCREASING TORQUE-SPACE DISTANCE FROM OC MOTION (INCREASINGLY DIFFERENT APPEARANCE). OC $=$ ORIGINAL COORDINATED MOTION. AVG $=$ AVERAGE PERCENT OF PARTICIPANTS OVER ALL VARIANTS. NOTE: PERCENTAGE RESOLUTION PER MOTION IS $1 \%$ DUE TO NUMBER OF PARTICIPANTS.

\begin{tabular}{l|c|c|c|} 
Motion & OC & Variants By Increasing Torque Distance & Avg \\
\hline Wave & 7 & $10,9,8,8,8,6,9,8,6,10,6,10$ & 8.17 \\
\hline Beckon & 9 & $11,7,10,11,7,11,8,10,9,6,10,11$ & 9.25 \\
\hline Shrug & 10 & $9,8,11,8,6,8,6,7,11,8,10,9$ & 8.42 \\
\hline Move Object & 8 & $8,6,7,6,10,8,7,11,8,11,9,8$ & 8.25 \\
\hline 1-Hand Bow & 6 & $8,8,7,7,9,11,6,10,11,11,11,7$ & 8.83 \\
\hline 2-Hand Bow & 10 & $9,7,6,8,8,8,10,9,11,9,8,10$ & 8.58 \\
\hline Scan & 10 & $9,11,8,10,7,11,7,9,10,8,7,10$ & 8.92 \\
\hline Look Around & 9 & $8,10,10,10,10,9,10,7,11,7,11,10$ & 9.42 \\
\hline Worship & 7 & $7,8,10,10,9,7,8,11,6,10,8,10$ & 8.67 \\
\hline Presentation & 11 & $8,7,8,7,10,7,10,10,7,11,10,11$ & 8.83 \\
\hline Air Guitar & 10 & $10,10,8,10,8,7,8,7,10,10,9,8$ & 8.75 \\
\hline Shucks & 10 & $11,9,11,10,10,8,6,9,8,8,10,10$ & 9.17 \\
\hline Bird & 8 & $10,9,10,10,7,6,10,9,10,7,9,10$ & 8.92 \\
\hline Stick 'em Up & 9 & $6,9,7,6,7,8,7,10,7,6,10,10$ & 7.75 \\
\hline Cradle & 7 & $11,6,7,10,10,11,9,10,8,10,9,7$ & 9.00 \\
\hline Call/Yell & 7 & $8,8,10,7,8,9,11,7,9,11,8,10$ & 8.83 \\
\hline Sneeze & 7 & $7,9,8,8,7,10,7,6,9,7,7,6$ & 7.58 \\
\hline Cover & 8 & $8,10,7,6,11,11,9,6,8,8,10,8$ & 8.50 \\
\hline Throw & 10 & $7,10,7,7,11,10,6,7,7,8,10,8$ & 8.17 \\
\hline Clap & 7 & $9,10,8,9,9,6,10,7,7,8,10,6$ & 8.25 \\
\hline & & &
\end{tabular}

resolution on the data in Table VIII is $1 \%$. From this data, we conclude that as the variants generated by the variance optimization look more different than the original motion, they maintain the human-like quality of that original motion.

By contrasting Tables VIII and IX the trend emerges. In Table VIII motions are not classified as more humanlike as they become more different in physical appearance from the OC input motion. However, by ordering motions according to decreasing STC, which according to the humanlike optimization yields more human-like motion, there is a very slight percent increase in responses of participants who labeled human-like motion in agreement with the STC trend (i.e. more human-like motion correlates to lower values of STC). Since the numbers in Table IX represent variants and one input $\mathrm{OC}$ version per motion, the variant motions also obey the rule that human-like motion has lower STC. This data supports H6, that the variance optimization is not strongly affecting the human-likeness of the input motion.

$63.75 \%$ of the 240 generated variants (first 12 variants, 20 motions) had percent of participant values (selecting them as the most human-like of the 13 options for each motion) which were greater than or equal to the input original coordinated motion. In fact, 5 of the 20 motions only had zero or one variant that was selected by fewer participants than the input OC motion to be the most human-like version. This means that using an input motion that is human-like 
TABLE IX

PERCENT OF PARTICIPANTS WHO SELECTED A VARIANT AS THE MOST HUMAN-LIKE FROM THE SET OF ORIGINAL COORDINATED MOTIONS AND THE FIRST 12 VARIANTS GENERATED BY THE VARIANCE OPTIMIZATION.

ALL VARIANTS AND MOTIONS (INCLUDING THE INPUT, ORIGINAL COORDINATED MOTIONS) HAVE THEIR RESPECTIVE PERCENTAGES SHOWN IN THE TABLE AND ARE ORDERED BY DECREASING STC (INCREASINGLY MORE HUMAN-LIKE). NOTE: PERCENTAGE RESOLUTION PER MOTION IS 1\% DUE TO NUMBER OF PARTICIPANTS.

\begin{tabular}{l|c|} 
Motion & Ordered By Decreasing STC \\
\hline Wave & $6,6,6,7,8,8,8,8,9,9,10,10,10$ \\
\hline Beckon & $6,7,7,8,9,9,10,10,10,11,11,11,11$ \\
\hline Shrug & $6,6,7,8,8,8,8,9,9,10,10,11,11$ \\
\hline Move Object & $6,6,7,7,8,8,8,8,8,9,10,11,11$ \\
\hline 1-Hand Bow & $6,6,7,7,7,8,8,9,10,11,11,11,11$ \\
\hline 2-Hand Bow & $6,7,8,8,8,8,9,9,9,10,10,10,11$ \\
\hline Scan & $7,7,7,8,8,9,9,10,10,10,10,11,11$ \\
\hline Look Around & $7,7,8,9,9,10,10,10,10,10,10,11,11$ \\
\hline Worship & $6,7,7,7,8,8,8,9,10,10,10,10,11$ \\
\hline Presentation & $7,7,7,7,8,8,10,10,10,10,11,11,11$ \\
\hline Air Guitar & $7,7,8,8,8,8,9,10,10,10,10,10,10$ \\
\hline Shucks & $6,8,8,8,9,9,10,10,10,10,10,11,11$ \\
\hline Bird & $6,7,7,8,9,9,9,10,10,10,10,10,10$ \\
\hline Stick 'em Up & $6,6,6,7,7,7,7,8,9,9,10,10,10$ \\
\hline Cradle & $6,7,7,7,8,9,9,10,10,10,10,11,11$ \\
\hline Call/Yell & $7,7,7,8,8,8,8,9,9,10,10,11,11$ \\
\hline Sneeze & $6,6,7,7,7,7,7,7,8,8,9,9,10$ \\
\hline Cover & $6,6,7,8,8,8,8,8,9,10,10,11,11$ \\
\hline Throw & $6,7,7,7,7,7,8,8,10,10,10,10,11$ \\
\hline Clap & $6,6,7,7,7,8,8,9,9,9,10,10,10$ \\
\hline
\end{tabular}

for the variance optimization will create variants that are subjectively deemed to be as human-like or more human-like as the input $63.75 \%$ of the time. Our variance optimization has a slight bias toward maintaining human-likeness.

\section{EXPERIMENT 5: VARIANCE OPTIMIZATION PRESERVES INTENT}

In the previous experiment we showed that the output of the variance optimization is still human-like, but we must also test that the intent of the original motion is preserved in the process of generating variants. According to $\mathrm{H} 7$, all variants should adhere to the definition of a true variant (i.e. they should be classified as the same motion type, gesture, or action as the original motion input to the variance optimization, from which the variants were created). In other words, when the robot moves using a variant, observers should perceive or label the robot intent to be the same as the intent they would perceive or label if the robot were moving using the input motion.

Experiment 5 demonstrates that the amount of variance produced by our algorithm is not so significant that it distorts variants to be labeled as gestures other than the original input motion.

\section{A. Experimental Design}

In this experiment, 153 participants were asked label a single variant of five different motions. The five motions were: shrug (i.e. "I don't know"), beckon, wave, bow, and point.
TABLE $X$

Percent CORReCtLy LABELED (\%) FOR 12 VARIANTS AND THE ORIGINAL MOTION OF FIVE DIFFERENT GESTURES. (10 SAMPLES PER VARIANT, 33 SAMPLES FOR EACH ORIGINAL MOTION). AVERAGE FOR ALL MOTION VARIANTS AS COMPARED TO THE ORIGINAL MOTION SHOWS THAT RECOGNITION DOES NOT DECREASE FOR GENERATED VARIANTS (I.E. VARIANTS THAT ARE CONSISTENTLY LABELED AS THE SAME MOTION TYPE AS THE ORIGINAL MOTION).

\begin{tabular}{l|c|c|c|c|c|} 
Motion & Beckon & Bow & Point & Shrug & Wave \\
\hline Variant 1 & 100 & 100 & 100 & 90 & 100 \\
\hline Variant 2 & 100 & 100 & 100 & 90 & 100 \\
\hline Variant 3 & 100 & 100 & 100 & 100 & 90 \\
\hline Variant 4 & 100 & 100 & 100 & 100 & 100 \\
\hline Variant 5 & 90 & 90 & 100 & 100 & 100 \\
\hline Variant 6 & 100 & 90 & 100 & 100 & 100 \\
\hline Variant 7 & 100 & 100 & 100 & 100 & 100 \\
\hline Variant 8 & 100 & 100 & 100 & 100 & 100 \\
\hline Variant 9 & 100 & 100 & 100 & 100 & 100 \\
\hline Variant 10 & 90 & 100 & 100 & 100 & 100 \\
\hline Variant 11 & 100 & 90 & 100 & 100 & 100 \\
\hline Variant 12 & 100 & 100 & 100 & 100 & 100 \\
\hline Var. Avg. & 98.3 & 97.5 & 100 & 98.3 & 99.2 \\
\hline Original & 93.9 & 93.9 & 100 & 87.9 & 100 \\
\hline
\end{tabular}

Participants watched videos of motions executed on the hardware so that the experiment could proceed more quickly, be more repeatable, and be well controlled. Participants watched the videos through web-based code, sequentially, and the experiment was conducted over the internet. After a video stopped playing, the screen blanked and prompted the user for a label. The next motion video appeared after the label was entered.

The order of the five motions was randomized, and each participant saw either the original motion version or one of 12 variant versions created using our algorithm. For Experiment 5, the 12 selected variant versions for each motion were approximately uniform selections based on torque distance from each other, so that a larger portion of the space of visually-different motions that can be produced by our algorithm could be covered in the analysis.

Thirty three participants saw the original motion version for a particular motion, leaving ten people who saw each variant version of that same motion. All orders and motions were randomized. No participant saw only original motion versions for all five motions.

\section{B. Results}

We use recognition as the metric for Experiment 5, to provide evidence that the variance optimization does not change motion intent. The task of the variance optimization is to produce a new version of the gesture that was given as the input motion. Thus, recognition (i.e. labeling of the variants with the same label as the original motion), was an appropriate metric to measure the success.

By comparing the bottom two rows in Table $\mathrm{X}$, we see that recognition rates are similarly high for the original motion and the variants. In four of five motions, percent 
correct recognition is slightly higher for the variants than for the original motion. Additionally, percent correctly labeled is never more than $0.8 \%$ worse for the variants generated with our algorithm. Therefore, we conclude that the variance optimization does a good job of preserving task intent.

\section{Experiment 6: Highly COnstrained Motion is LESS HUMAN-LIKE}

The final three experiments test the effect of constraints on either human-likeness or variance. From preceding experiments, we have demonstrated that the serial concatenation of the human-like and variance optimizations have successfully generated human-like variants of the original input motion. Since we claim that we can produce human-like, varied motion for robots, it is important to test how constraints influence our results, so that motion can be purposeful, human-like, and varied.

Experiment 6 is an analysis of human-likeness in the presence of increasing numbers of Cartesian constraints. According to hypothesis $\mathrm{H} 8$, as the number of constraints increases, SC and TC should decrease slightly for the human-like optimized motion after Cartesian constraints are applied. Hypothesis $\mathrm{H} 8$ is theorized as such because the dynamically consistent generalized inverse, which is essential in the projection that cancels constraint-affecting torques, does not account for human-likeness. Human-likeness should be affected very little when number of simultaneouslyapplied constraints is small. However, as the number of simultaneously-applied constraints increases, the null space decreases, which means that there is less redundancy with which to move in a human-like manner. Thus, the resultant motion in the presence of high quantities of external constraints will be less human-like.

\section{A. Experimental Design}

We designed Experiment 6 to test $\mathrm{H} 8$ and the interaction between the human-like optimization and external constraints. In previous experiments, participants were recruited to test whether spatially and temporally-optimized motion appeared more human-like than other types of motions. SC and TC were presented as a metric for human-like motion to find a quantitative measure for humans' subjective perception with respect to human-likeness in motion. Since hypotheses H1-H5 confirmed that the human-like optimization increases the human-likeness of motions, participants no longer need to be recruited to determine which motions are more humanlike. Rather, Equation 3 can be used as a metric to tell which motions are more human-like (i.e. SC and TC closer to zero, more coordinated, are more human-like motion).

The intuition behind the design of this experiment is that if the benefits of lower SC and TC values are preserved in the presence of constraints, then motion with constraints remains more coordinated (and therefore, more human-like) than motion that excludes the human-like optimization.

We compare four types of motion:

- Human Reference Condition (HRC): This is the baseline comparison data set for SC and TC; comprised of the original human motion and mimicked human motion data sets from Experiment 1. For this data set, SC and TC are evaluated on human models.

- With Human-like Optimization (WHO): The HRC data set after the human-like optimization (without constraints). For this data set, SC and TC are evaluated on a robot model.

- With Constraints Only (WCO): The HRC data set with constraints applied (without the human-like optimization). For this data set, SC and TC are evaluated on a robot model.

- With Human-like Optimization and Constraints (WHC): The HRC data set after both the human-like optimization and applied constraints. For this data set, SC and $\mathrm{TC}$ are evaluated on a robot model.

The original human and mimicked human data sets were appropriate for the reference set because they represent human motion and were constrained by mimicking so the variance of SC and TC should be quite small. In Experiment 1 , we showed that mimicking is an appropriate constraining method when motion comparisons must be made with respect to human-likeness.

To create the HRC data set, the motion capture trajectories from Experiment 1 for the original human and mimicked human $(\mathrm{OH}$ and $\mathrm{MH})$ data were optimally projected onto human models as described in Experiment 2. Then the two halves of the right-hand side of Equation 3 were evaluated for trajectories for all 20 motions to create a distribution for $\mathrm{SC}$ and TC.

To create the remaining three data sets, the first step is to optimally project the $\mathrm{OH}$ and $\mathrm{MH}$ trajectories onto the Simon model. Then for the human-like set (WHO), we perform the human-like optimization, and then evaluate the two halves of the right-hand side of Equation 3 for SC and TC. The humanlike and constraints (WHC) data set was created by taking the WHO data set and applying external constraints randomly before evaluating SC and TC. Finally, the constraints only (WCO) data set was created by optimally projecting the $\mathrm{OH}$ and $\mathrm{MH}$ data onto the Simon model, applying the same set of external constraints as for the WHC data set, and then evaluating $\mathrm{SC}$ and $\mathrm{TC}$.

Specifically, we hypothesized that the data after the human-like optimization only (WHO) would have the lowest SC and TC values, followed by human-optimized and constrained data (WHC), and then the data with constraints only (WCO) because constraints are expected to decrease humanlikeness on average and without the optimization to reduce STC, SC and TC are likely to be higher. If the results show this order, then we will have provided evidence in support of hypothesis H8. The results should also show that increasing constraints for the consrained data sets (i.e. WHC and WCO) should increase both SC and TC.

To strengthen the results, the number of simultaneously applied constraints varied from 1 to 5 for each of the 20 motions to help demonstrate that as number of constraints increases SC and TC decrease. For the purpose of data analysis, number of simultaneously applied constraints was 
assigned so that the same four motions only ever had one constraint applied, a different set of four only ever had two simultaneously-applied constraints, a different set of four only ever had three, etc.

H8 is independent of constraint type, and therefore, Cartesian position, orientation, look-at, or point-at were the types from which random selection was made. Each constraint was applied for a time duration of $5 \%$ of the total length of the trajectory. In cases where more than one constraint was simultaneously applied, all constraints were applied during the same time period and for the same duration. Constraint type, duration, number simultaneously applied, and time period of application were identical between the constrained data groups (WCO and WHC).

\section{B. Results}

On a motion-by-motion basis, treating all the data (HRC, WHC, WCO, WHO) as if it comes from a single distribution, F-tests for both SC and TC for 20 of 20 motions yield $\mathrm{p}<0.05$, which indicates that at least one of these groups is statistically different than the rest of the data. Subsequent pairwise F-tests determined that 0 of 20 motions displayed statistical significance $(\mathrm{p}<0.05)$ for HRC vs. WHO (both SC and TC); 20 of 20 motions displayed statistical significance $(\mathrm{p}<0.05)$ for HRC vs. WCO (both SC and TC); 2 of 20 motions (SC) and 3 of 20 motions (TC) displayed statistical significance $(\mathrm{p}<0.05)$ for HRC vs. WHC. These results are consistent with Experiment 1 because WCO is a constrained form of retargeted human motion, similar to the retargeted motion (i.e. OR and MR) from that experiment, which also found statistical significance between human and retargeted human motion (i.e. the $\mathrm{OH}$ and $\mathrm{MH}$ vs. $\mathrm{OR}$ and MR data sets in Experiment 1). There is no reason to believe that constraints will improve human-likeness. The five instances of constrained and human-optimized (WHC) motion that were statistically different all occurred for motions with five simultaneously-applied constraints.

This begins to support H8, and can also be seen in Figures 9 and 10. As more constraints were added in both the constrained motion (WHC and WCO) groups, SC and TC increased, which means that motion became less coordinated. In the graphs each group shows all 20 motions ordered from most constrained at the top, and least constrained at the bottom. As stated in the previous paragraph all of the WCO motions were significantly less coordinated, which can be seen in their mean SC and TC values. Additionally, with more constraints added to the motion, the SC and TC metrics began to exhibit noticeably larger differences between the WHC group and each of the reference condition and the human-like optimized only group. When number of constraints increased to 5 simultaneously applied, statistical difference was observed in all 5 instances. The overall values of SC and TC for the motion group that only had constraints increased as number of simultaneously-applied constraints increased (from approximately 0.27 with one constraint up to 0.32 with five constraints for SC and TC).

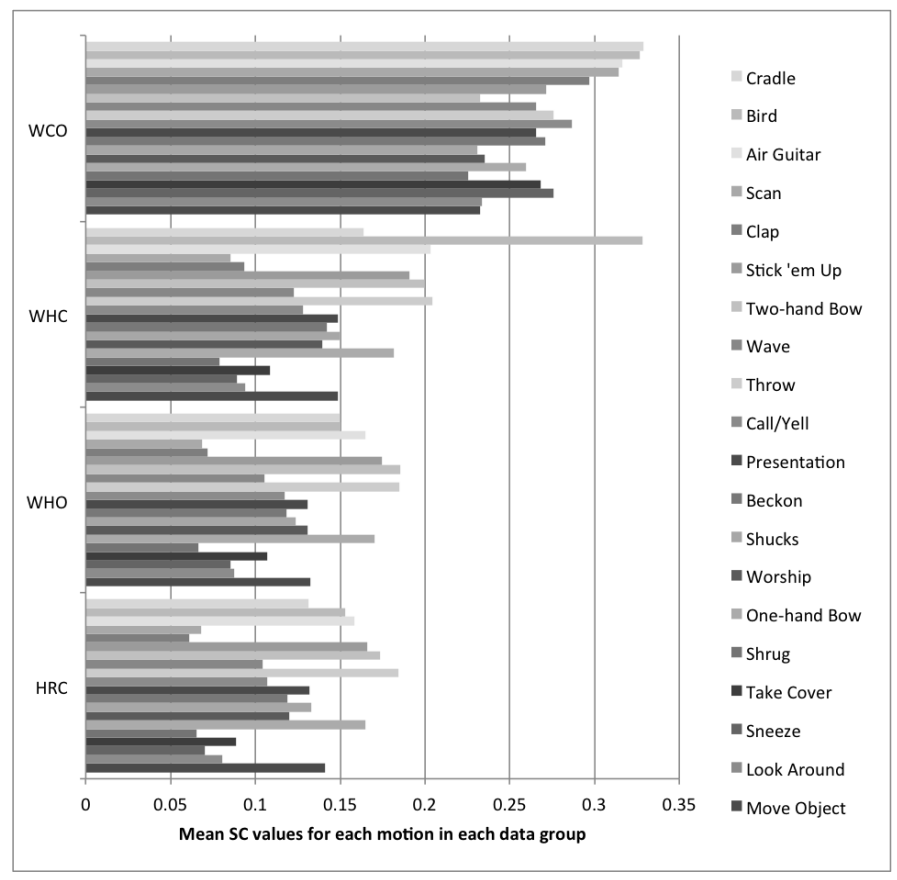

Fig. 9. Mean SC values for three data groups (WHO, WHC, WCO) as calculated by the respective parts of Equation 3. Motions are grouped according to number of simultaneously applied constraints (i.e. first four motions (Cradle-Scan) have 5 constraints, then Clap-Wave have 4 simultaneously applied constraints, etc.). $\mathrm{HRC}=\mathrm{OH}+\mathrm{MH}$ human data from Experiment 1; $\mathrm{WHO}=\mathrm{HRC}$ projected to the robot model with SC and TC calculated after the human-like optimization; $\mathrm{WHC}=\mathrm{HRC}$ projected onto the robot with both the human-like optimization and applied constraints; WCO $=$ HRC on the robot model with only constraints applied.

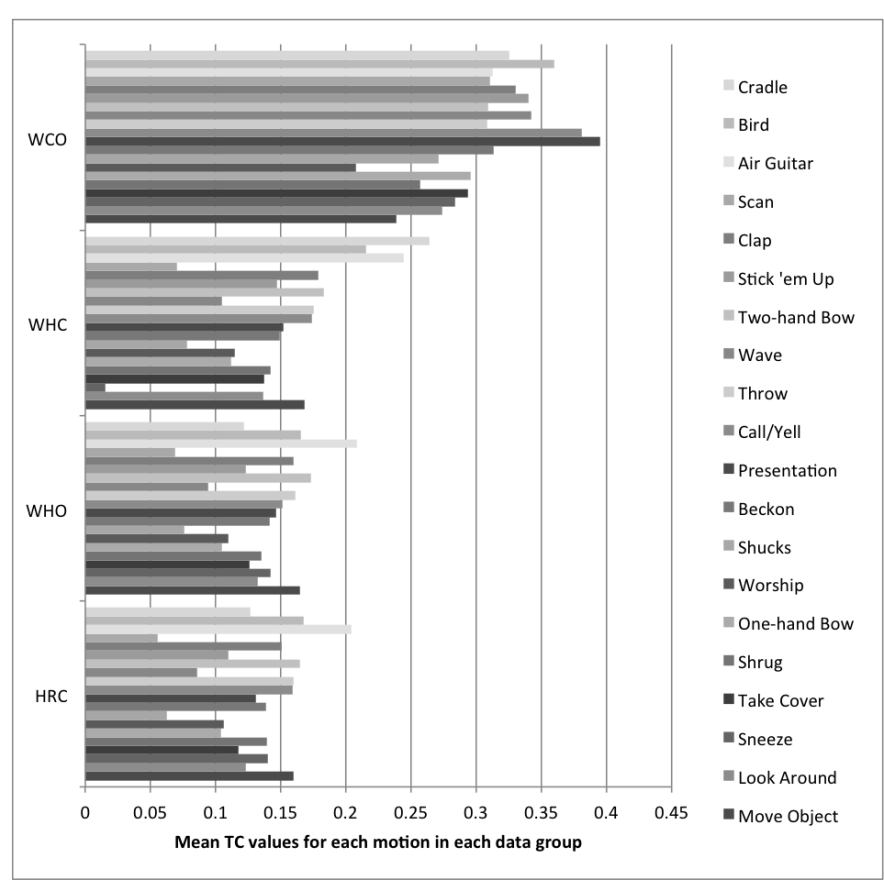

Fig. 10. This is the analogous graph to Figure 9, for mean TC values, which show similar trends as SC.

Furthermore, of the three data groups that were tested (WHO, WHC, and WCO), the set of motions that only 
underwent the human-like optimization (i.e. WHO) was the most coordinated motion of all three, as seen by the lowest SC and TC values in Fig. 9 and 10. These values are also closest to the human reference condition (i.e. HRC) set values, which supports $\mathrm{H} 8$ that adding constraints begins to limit human-likeness.

\section{EXPERIMENT 7: Highly Constrained Motion HAS LESS VARIANCE}

Experiment 6 showed that as number of constraints increased, human-likeness decreased. However, we still need to study the effect of constraints on variance. Experiment 7 represents an analysis of variance in the presence of increasing the number of constraints. According to hypothesis H9, as the number of constraints increases, the variants should deviate less from their average value. No general claims can be made about the amount of variance in the variants relative to each other or relative to the motion input to the variance optimization because the degree to which these variants are similar to the input or to each other strongly depends on the selected constraints. Thus, as number of constraints increases, deviation of the variants from each other or the input motion could increase or decrease because the appearance of the variants is a strong function of the selected constraints. Rather, hypothesis H9 is based upon a smaller null space as number of constraints increases. Since the variants are projected into the null space of the constraints in our algorithm, more constraint-disrupting torque is canceled, and therefore, less torque actuates the variants.

Variance should be affected very little when number of simultaneously-applied constraints is small. Consider the simple example of one joint position constraint that lasts for the duration of the entire motion. Compared to the situation with zero constraints, all torque in that one degreeof-freedom will be canceled and cumulative variance for the motion with one constraint will be less. When considering the extreme case where all DOFs are constrained by joint position for the duration of the entire motion, clearly variance become zero and motion ceases. Thus, the resultant motion in the presence of high quantities of constraints will be less varied.

\section{A. Experimental Design}

We designed Experiment 7 to test $\mathrm{H} 9$ and the interaction between the variance optimization and constraints. However, since the case of joint position constraints is clear from the presented example, these particular constraints were excluded from the experiment. All other constraints were included: joint velocity, joint torque, point, orientation, pointat, and look-at.

In Experiment 7, three types of motion exist:

- Robot Reference Condition (RRC): The twenty robot motions from Experiment 1, including one-handed (left or right arm) and two-handed gestures (symmetric).

- With Variance Only (WVO): The RRC data set after the variance optimization (without constraints).
- With Variance and Constraints (WVC): The WVO data set with applied constraints.

For the data set with constraints applied (i.e. WVC), a window of $5 \%$ of the motion length was chosen at random and up to 10 randomly selected constraints were applied during this window (i.e. each constraint was applied for a time duration of $5 \%$ of the total length of the trajectory). In cases where more than one constraint was simultaneously applied, all constraints were applied during the same time period and for the same duration. Fifty different variants were generated with fifty unique windows for each of twenty motions (i.e. 1000 data points).

\section{B. Results}

Variance was calculated for each motion and the data is arranged in Fig. 11 according to number of applied constraints. Figure 11 shows a distinct trend for constraints. As more constraints were added in both the WB group, average variance decreased, which is consistent with H9. There is a significant drop in variance above four constraints, and the constrained motions use only one-third of their joint angle ranges.

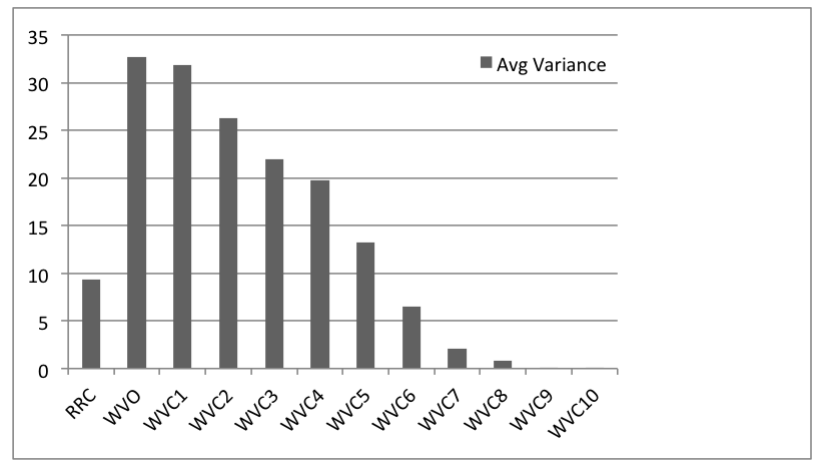

Fig. 11. Mean variance for unconstrained (WVO) and constrained motion (WVC) ordered by number of constraints. The number follow ing WVC indicates the number of constraints applied. Constraints were applied over a 5\% trajectory length for all constrained data. Each row in the table is an average of 1000 data points (50 variants of 20 different motions; 1 unique constraint window for each variant). Averages taken over all DOFs. Results are expressed as a percentage of total DOF range (limit to limit). E.g. average variance of 50 would mean that the average DOF was utilizing $50 \%$ of its joint angle range for all variants.

The data in Fig. 11 quantifies the average performance of the variance optimization. The ratio of WVO to RRC shows that the variance optimization on average produces motion that uses 3.5 times more of the joint angle ranges than in the original motions. This is consistent with the algorithm because we are adding biased torque noise in low cost dimensions, which, on average, results in an increased amount of motion in the variants. The ratio of 3.5 is also a good measure of "noticeablility" because it might be unintuitive to believe that injecting a small amount of torque noise would produce noticeably-different variants. The $350 \%$ increase in use of joint angle ranges is a good indicator that variants will be noticeably-different.

After 10 constraints are applied, nearly all motion is lost in the average degree-of-freedom. According to the data in Fig. 
11 , anything more than seven constraints applied causes very little use of joint angle ranges (i.e. little motion, little variance). Humanoid robots are very similar to humans, which cannot balance seven simultaneous tasks without sacrificing the lowest priority tasks. Therefore, in a way, this is further evidence that the constrained variants are human-like.

\section{EXPERIMENT 8: CONSTRAINED Body PARTS EXHIBIT LESS VARIANCE}

In Experiments 6 and 7, we examined the effects of increasing the number of constraints. In our final experiment, we now evaluate variance as a function of proximity to the constraint. Although it might be intuitive that constrained body parts will move less, we provide Experiment 8 to demonstrate that constraints work as designed. The projection into the null-space to satisfy Cartesian constraints should affect the variance induced by the variance optimization more as proximity to the constraint increases. To support H10, Experiment 8 was designed to analyze variance in the presence of multiple constraints. Cartesian constraints on the robot's body force the robot architecture to satisfy some geometry: point, line, or plane, which causes the constrained body parts to move less in Cartesian space. Observations such as these motivated hypothesis H10. Robot body parts that are connected in a series chain to a constrained body part will also exhibit less variance. These effects result from the null space because the null space is smaller closer to kinematically constrained body parts. We test the null space to provide quantitative support for hypothesis H10.

\section{A. Experimental Design}

One point constraint and one orientation constraint were applied to the robot's left hand for the entire duration of a shrug trajectory to constrain the hand so that a cup of water did not spill. Deviation in the left arm was then analyzed with respect to the original motion (Equation 12) and average inter-variant variance (Equation 13) for two conditions: with and without constraints. Comparable numbers for constrained and unconstrained conditions on a per-DOF basis would support the null hypothesis that constraint projection does not adversely affect variance. This analysis was specifically performed on the arm closest to the constraint because part of hypothesis $\mathrm{H} 10$ suggests that variance decreases closer to the constrained location on the architecture.

$$
\sigma_{h}^{2}=\frac{1}{K} \frac{1}{N} \sum_{i=1}^{K}\left(\bar{q}-q_{i}\right)^{T}\left(\bar{q}-q_{i}\right)
$$

where,

$$
\begin{aligned}
& \sigma_{h}{ }^{2}=\text { average square deviation of DOF } h \text { with respect to } \\
& \text { original, input motion } \\
& q_{i}=\text { the joint angle trajectory of DOF } h \text { from variant } i \\
& \text { as a vector } \\
& \bar{q}=\text { the joint angle trajectory of DOF } h \text { from the original, } \\
& \text { input motion as a vector } \\
& K=\text { number of variants used in the analysis } \\
& N=\text { number of time samples in given reference trajectory }
\end{aligned}
$$

TABLE XI

AVERAGE VARIANCE (STANDARD DEVIATION) OF JOINT ANGLES FOR ALL LEFT ARM DOFS (IN SQUARE DEGREES). DEVIATION FROM ORIGINAL MOTION AND INTER-VARIANT VARIANCE (DEVIATION BETWEEN GENERATED VARIANTS; LABELED “BETWEEN”) GIVEN FOR CONDITIONS OF CONSTRAINED AND UNCONSTRAINED MOTION.

AVERAGES TAKEN USING THE FIRST 12 MOTIONS GENERATED BY THE VARIANCE ALGORITHM FOR A SHRUG (“I DON'T KNOW”) GESTURE WHILE CONSTRAINED WITH THE LEFT HAND HOLDING A CUP.

\begin{tabular}{l|c|c|c|c|} 
& \multicolumn{2}{|c|}{ Unconstrained } & \multicolumn{2}{c}{ Constrained } \\
DOF & w.r.t. orig & between & w.r.t. orig & between \\
\hline shoulder X & $312(241)$ & $396(197)$ & $116(55)$ & $354(121)$ \\
\hline shoulder Z & $305(190)$ & $213(209)$ & $271(123)$ & $133(58)$ \\
\hline shoulder Y & $314(139)$ & $457(212)$ & $298(286)$ & $599(342)$ \\
\hline elbow X & $602(229)$ & $954(799)$ & $581(119)$ & $846(837)$ \\
\hline wrist Y & $442(251)$ & $941(642)$ & $560(175)$ & $1132(899)$ \\
\hline wrist X & $279(281)$ & $260(317)$ & $22(16)$ & $189(119)$ \\
\hline wrist Z & $171(192)$ & $194(236)$ & $44(37)$ & $124(63)$ \\
\hline
\end{tabular}

$$
A I V V_{h}=\frac{1}{(K-1)^{2}} \frac{1}{N} \sum_{\substack{i=1 \\ i \neq j}}^{K} \sum_{j=1}^{K}\left(q_{j}-q_{i}\right)^{T}\left(q_{j}-q_{i}\right)
$$

\section{B. Results}

To measure variance in the presence of constraints, the variance of the left arm DOFs provided more insight into how constraints affect the motion because the left arm was closer to the constraint in the task. We first constrained the original motion, and then we used the original motion torques as the mean to compute the variance (Equation 12). The results are shown in Table XI.

As expected, physical constraints reduce variance overall, as shown in Table XI. This was especially true closer to the actual constrained DOF, which for the cup constraint occurred in the wrist for the $\mathrm{x}$ and $\mathrm{y}$ degrees-of-freedom. This effect was also evident in the calculations that reference the other variants. However, average variance between variants was still large for the left arm DOFs.

By comparing data for constrained and unconstrained, the trend was that constrained deviation was smaller when further from the wrist (i.e. point of constraint application). The constrained numbers approach unconstrained variance further from the wrist, which indicates the impact of constraints upon variance. From our analysis we concluded that the variance optimization also produces variance in constrained motion, provided that a null space for the constraints exists. H10 is supported since the data clearly indicates that variance is limited near the point of application of a constraint. As more constraints are added, less variance can be expected.

\section{DISCUSSION}

We have presented an algorithm for generating humanlike motion on robots in an autonomous way. Our underlying assumptions in this work are that: (a) the robot has controllable, independent actuators; (b) at least one input motion 
exists; and (c) the robot receives the constraints it must satisfy through sensors or a higher-level, decision-making algorithm.

Thus, given these assumptions, our algorithm is applicable to any robot that needs or wants to move in a more life-like manner. In this work, we have taken significant steps to show human-likeness, but it is likely that the algorithm will work for any biologically-inspired robot that would have muscles if it were alive, because animal motion has also been shown to exhibit spatio-temporal coordination due to their musculature [13].

The three components of our process are:

1) Spatio-temporal coordination: We optimize motion with respect to spatio-temporal correspondence, which emulates the coordinated effects of human joints that are connected by muscles.

2) Variance: We add variance to avoid repetitive motion by exploiting redundant and underutilized spaces of the input motion, which creates multiple motions from a single input. Possessing multiple versions of the same motion gives the robot the ability to choose a collisionfree path when faced with obstacles.

3) Constraints: We maintain the robot's ability to interact with it's world by providing it the ability to satisfy constraints.

The extent of the work thus far has focused on validating these three components of our pipeline for generating humanlike motion in a series of eight experiments that we have presented here.

\section{A. Summary of Findings}

Experiments 1, 2 and 3 validated the first component of our algorithms, the human-like optimization of Spatiotemporal coordination. In experiment 1 we learned that the humanlike optimization can make retargeted motion more natural looking and easier for people to identify intent. Experiment 2 additionally showed that applying our optimization to motion that is already human-like does not alter it significantly; that the human-likeness is left in tact. And experiment 3 showed that even when we intentionally eliminate some information in the motion signal (by downsampling), the optimization process is still able to recover that information to produce a human-like motion. Thus we conclude from this collection of experiments that increasing spatio-temporal correspondence of distributed actuators synthesizes more human-like motion.

Next in the pipeline is the ability to produce multiple variants of a single input motion. Remember that the motivation for doing this is that humans rarely if ever repeat a motion exactly the same way twice, thus the ability to add variance in a human-like way is an important step in our process. In experiment 4 we validated that the output of our variance optimization is still human-like. And in experiment 5 we demonstrated the amount of variance produced by our algorithm and validated that it does not distort the input motion so much as to hinder people's ability to recognize the motion's intent.
The final piece in the pipeline is a practical one for robots that need to generate a particular motion in the face of environmental or task constraints (e.g., a target location/orientation of a particular joint, a look-at constraint during the motion, etc.). In experiment 6 we showed that as more of these constraints are added the human-likeness of the resulting motion is decreased. In experiments 7 and 8 we analyzed variance in the presence of increasing constraints, and at a joint level as a function of proximity to the constraint. Thus, as expected as the number of constraints are increased for a motion, then the ability for our algorithm to produce human-like varied motion decreases. But we found that in the presence of less than 5 simultaneous constraints our approach is successful in generating human-like motion.

\section{B. Implications for Human-Robot Interaction}

Our future work will explore the benefits that humanlike motion has on human-robot interaction. For example, we have shown that human-like motion produces improved motion clarity and better recognition for human partners, but what are the distinct benefits that both partners derive because humans are better able to tell what the robot is doing? Will collaboration progress more quickly? Will joint action be smoother with fewer errors? Will human enjoyment and satisfaction with the robot and the interaction improve? Will coordination between partners be more accurate and efficient? Will interactions achieve higher success rates when robot motion is more human-like? Will humans interact with robots longer or feel more engaged in interactions because the robot exhibits human-like motion? These and many other questions are the higher-level goals of our work with human-like motion. Demonstrations of these benefits and many others will become the motivation for others to equip their robots with our algorithm to autonomously generate human-like motion. Addressing these benefits will also enable others' to decide where, when, and how exactly to use the algorithm that we have outlined herein.

We have shown that our algorithm produces robot motion that human partners can more easily identify due to the human-likeness imparted by our optimization. And given that this human-likeness in motion is preserved in the presence of variance and constraints, robots that collaborate with human partners will more clearly indicate their intent to their partners if our algorithm is used to synthesize robot motion.

This paper revolutionizes trajectory design for robots since databases of numerous examples of different motions and complex dynamic equations of motion are no longer required to generate variance. Although higher numbers of constraints will restrict variance and human-likeness in robot motion, one exemplar of each motion type (e.g. one wave, one shrug, one reach) is all that is required for the robot to perform an infinite number of motions similar to the exemplar. That exemplar trajectory can even be observed to avoid storage issues. Furthermore, we have shown that imperfect observation of such trajectories is sufficient to still create humanlike robot motion because our human-like optimization can recover information lost in the trajectory. 
The challenges in using this algorithm are few. The robot requires a torque trajectory, which means that if the given trajectory is not in torque-space, inverse dynamics might need to be performed on the given trajectory (which would require a model of the agent from which the exemplar trajectory was obtained). The algorithm does not exclude the use of human motion capture data for the exemplar trajectory, but use of such data requires a projection algorithm between the human and robot models to provide the input trajectory in proper form.

We also contribute spatial and temporal correspondence as a metric for human-like motion for robots. The impact of this contribution is that a consistent baseline, which is independent of kinematics and dynamics, is presented for motion comparison. The independence of this metric means that any two agents that have degrees-of-freedom can have motion compared regardless of domain (e.g. cartoon, puppet, robot, human, virtual character). Our metric is the first metric that can be used to either evaluate or synthesize human-like motion. The additional advantages of our metric are that it is objective, does not require dynamic simulations, and it is not observer- or heavily data-dependent.

Our experiments have also shown that to adequately compare human motion data objectively, it must be constrained. We have done so using mimicking, which reduces the variance of the range of motions humans can produce so that data is comparable between different humans. Constraining motion via mimicking also restricts the variance induced due to difference in kinematics and dynamics for a human population, which further facilitates scientific inquiry and data analysis.

\section{CONCLUSION}

We presented an algorithm that produces human-like variants of an input motion. This algorithm is made functional for real-world tasks by including a projection that allows the robot to satisfy constraints such as looking at, grabbing, or pointing at objects. Similarly, the algorithm allows robots to synchronize to their partners, provided that human partners' actions can be observed. Through a series of eight experiments, we show that human-like robot motion improves recognition accuracy of robot motions, so that social partners can more easily and accurately tell what the robot is doing. This increased clarity in motion can improve collaboration fluidity and minimize distractions due to awkward robot motion. Variance was added to the human-like motion so that human-likeness would be further improved and robot motion would be less repetitive.

\section{FUNDING}

This work was supported by Office of Naval Research Young Investigator Grant [grant number N000140810842]; and National Science Foundation CAREER Grant [grant number IIS-1032254].

\section{REFERENCES}

[1] T. Asfour, K. Berns, and R. Dillmann. The humanoid robot ARMAR: Design and control. 1st IEEE-RAS Intl. Conference on Humanoid Robots, September 2000.

[2] T. Asfour, A. Ude, K. Berns, and R. Dillmann. Control of ARMAR for the realization of anthropomorphic motion patterns. Second Intl. Conf. on Humanoid Robots, pages 22-24, 2001.

[3] E. Bicho, W. Erlhagen, L. Louro, and E. C. e Silva. Neurocognitive mechanisms of decision making in joint action: a human-robot interaction study. Hum Movement Sci, 2011.

[4] A. Dasgupta and Y. Nakamura. Making feasible walking motion of humanoid robots from human motion capture data. In Proceedings of the 1999 IEEE Intl. Conference on Robotics and Automation, pages 1044-1049, May 1999.

[5] B. Duffy. Anthropomorphism and the social robot. Robot Auton Syst, 42(3-4):177-190, 2003.

[6] T. Flash and N. Hogan. The coordination of arm movements: An experimentally confirmed mathematical model. The Journal of Neuroscience, 5(7):1688-1703, July 1985.

[7] T. Fong, I. Nourbakhsh, and K. Dautenhahn. A survey of socially interactive robots. Robotics and Autonomous Systems, 42(3-4):143166, 2003.

[8] T. Fukuda, R. Michelini, V. Potkonjak, S. TZAFESTAS, K. Valavanis, and M. Vukobratovic. How far away is "artificial man". Robotics Automation Magazine, IEEE, 8(1):66-73, 2001.

[9] M. Gielniak and A. L. Thomaz. Anticipation in robot motion. ROMAN, 2011.

[10] M. Giese and T. Poggio. Morphable models for the analysis and synthesis of complex motion pattern. Intl. Journal of Computer Vision, February 2000.

[11] M. Gleicher. Retargeting motion to new characters. In Proceedings of SIGGRAPH, pages 33-42, July 1998.

[12] K. Harada, K. Hauser, T. Bretl, and J. Latombe. Natural motion generation for humanoid robots. In Proceedings of the 2006 IEEE/RSJ Intl. Conference on Intelligent Robots and Systems, pages 833-839, October 2006.

[13] J. Hayfron-Acquah, M. S. Nixon, and J. N. Carter. Recognising human and animal movement by symmetry. In International Conference on Image Processing, 2001.

[14] O. Jenkins and M. Mataric. A spatiotemporal extension to isomap nonlinear dimension reduction. Technical Report CRES-04-003, University of Southern California Center for Robotics and Embedded Systems, 2004.

[15] A. Kolmogorov. Entropy per unit time as a metric invariant of automorphisms. Doklady Akademii Nauk SSSR, 124:754-755, 1959.

[16] K. Kondo. Inverse kinematics of a human arm. Technical Report CS-TR-94-1508, Stanford University, 1994.

[17] B. Lay, W. Sparrow, K. Hughes, and N. ODwyer. Practice effects on coordination and control, metabolic energy expenditure, and muscle activation. Human Movement Science, 21(56):807 - 830, 2002. ice:title ${ }_{i}$ Current issues in motor control and coordination ${ }_{i} /$ ce:title $_{i}$.

[18] J. Lee, J. Chai, P. Reitsma, J. Hodgins, and N. Pollard. Interactive control of avatars animated with human motion data. In Proceedings of the 29th Annual Conference on Computer Graphics and Interactive Techniques, pages 491-500, 2002.

[19] F. L. Lewis and V. L. Syrmos. Optimal Control. Wiley-Interscience, 1995.

[20] C. Liu and Z. Popovic. Synthesis of complex dynamic character motion from simple animations. In Proceedings of the 29th Annual Intl. Conference on Computer Graphics and Interactive Techniques, pages 408-416, 2002.

[21] T. Moeslund and E. Granum. A survey of computer vision-based human motion capture. CVIU, pages 231-268, 2001.

[22] D. Oziem, N. Campbell, C. Dalton, D. Gibson, and B. Thomas. Combining sampling and autoregression for motion synthesis. In Proceedings of the Computer Graphics Intl. Conference, pages 510513, June 2004.

[23] R. Playter. Physics-based simulations of running using motion capture. SIGGRAPH 2000 Course Notes, Course \#33, 2000.

[24] Z. Popovic and A. P. Witkin. Physically based motion transformation. In Proceedings of SIGGRAPH 99. Computer Graphics Proceedings, Annual Conference Series, pages 11-20, 1999. 
[25] M. Prokopenko, V. Gerasimov, and I. Tanev. Measuring spatiotemporal coordination in a modular robotic system. In L. M. Rocha, L. S. Yaeger, M. A. Bedau, D. Floreano, R. L. Goldstone, and A. Vespignani, editors, Artificial Life X: Proceedings of the Tenth International Conference on the Simulation and Synthesis of Living Systems, pages 185-191. MIT Press, 3-7 June 2006.

[26] P. Reitsma and N. Pollard. Perceptual metrics for character animation: Sensitivity to errors in ballistic motion. ACM Trans. Graph., 22(3):537-542, 2003.

[27] L. Ren, A. P. A. A. Efros, J. K. Hodgins, and J. M. Rehg. A datadriven approach to quantifying natural human motion. ACM Trans. Graph., 24(3):1090-1097, 2005.

[28] N. Sebanz, H. Bekkering, and G. Knoblich. Joint action: bodies and minds moving together. Trends Cogn Sci, 10(2):70-76, 2006.

[29] L. Sentis and O. Khatib. A whole-body control framework for humanoids operating in human environments. IEEE Intl. Conf. on Robotics and Automation (ICRA)., pages 2641-2648, May 2006.

[30] Y. Song, L. Goncalves, and P. Perona. Unsupervised learning of human motion. IEEE Trans. on Pattern Analysis and Machine Intelligence, 25(7):814-827, July 2003.

[31] R. Tomovic, G. Bekey, and W. Karplus. A strategy for grasp synthesis with multifingered robot hands. In Proceedings IEEE Intl. Conf. on Robotics and Automation, pages 83-89, 1987.

[32] D. Wu. Ad hoc meta-analysis of perceived error in physically simulated characters. Game Developers Convention. Microsoft Game Studios, 2009.

[33] K. Yamane, J. Kuffner, and J. Hodgins. Synthesizing animations of human manipulation tasks. ACM Transactions on Graphics, 23(3):532-539, 2004. 